\title{
Efficacy and Safety of Curcumin Supplement on Improvement of Insulin Resistance in People with Type 2 Diabetes Mellitus: A Systematic Review and Meta-Analysis of Randomized Controlled Trials
}

\author{
Tianqing Zhang, ${ }^{1}$ Qi He $\mathbb{D}^{2},{ }^{2}$ Yao Liu, ${ }^{1}$ Zhenrong Chen, ${ }^{1}$ and Hengjing Hu $\mathbb{C}^{1,3}$ \\ ${ }^{1}$ The First Affiliated Hospital, Department of Cardiovascular Medicine, Hengyang Medical School, University of South China, \\ Hengyang, Hunan Province, China \\ ${ }^{2}$ People's Hospital of Ningxiang City, Ningxiang City, Hunan Province, China \\ ${ }^{3}$ Institute of Cardiovascular Disease and Key Lab for Arteriosclerology of Hunan Province, University of South China, Hengyang, \\ Hunan, China \\ Correspondence should be addressed to Hengjing Hu; bestmanhhj123@163.com
}

Received 6 June 2021; Accepted 20 July 2021; Published 25 August 2021

Academic Editor: Denise Brentan Silva

Copyright (C) 2021 Tianqing Zhang et al. This is an open access article distributed under the Creative Commons Attribution License, which permits unrestricted use, distribution, and reproduction in any medium, provided the original work is properly cited.

\begin{abstract}
Background. Diabetes is a major public health concern. In addition, there is some evidence to support curcumin as part of a diabetes treatment program. Methods. Data from randomized controlled trials were obtained to assess the effects of curcumin versus placebo or western medicine in patients with type 2 diabetes mellitus (T2DM). The study's registration number is CRD42018089528. The primary outcomes included homeostasis model assessment-insulin resistance (HOMA-IR), glycosylated hemoglobin (HbAlc), total cholesterol (TC), and triglyceride (TG). Results. Four trials involving 453 patients were included. The HOMA-IR of curcumin group is lower in Asia (WMD: $-2.41,95 \% \mathrm{CI}:-4.44$ to $-0.39, P=0.02$ ) and the Middle East subgroups (WMD: $-0.60,95 \% \mathrm{CI}:-0.74$ to $-0.46, P<0.00001$ ). The HbAlc in the curcumin group is lower than that in the control group (WMD: $-0.69 ; 95 \% \mathrm{CI}:-0.91,-0.48 ; P<0.0001$ ). The TC and TG levels of the curcumin group are lower in the Asia subgroup (TC: WMD: $-23.45,95 \%$ CI: -40.04 to $-6.84, P=0.006$; TG: WMD: $-54.14,95 \%$ CI: -95.71 to $-12.57, P=0.01$ ), while in the Middle East the difference was of not statistically significant (TC: WMD: $22.91,95 \% \mathrm{CI}:-16.94$ to $62.75, P=0.26$; TG: WMD: $-4.56,95 \%$ CI: -19.28 to $10.16, P=0.54$ ). Conclusion. Based on the current evidence, curcumin may assist in improving the insulin resistance, glycemic control, and decreased TG and TC in patients with T2DM.
\end{abstract}

\section{Introduction}

As a serious metabolic disease, diabetes affects about $5 \%$ of the world's people. Epidemiological data show that the number of people with diabetes is expected to increase dramatically to 592 million by 2035 [1]. 12\% of global health expenditure is spent annually on diabetes and its complications [2]. Diabetes is divided into different types, wherein type 1 and type 2 diabetes accounted for more than $90 \%$ of all cases. Among these types of diabetes, type 2 diabetes mellitus (T2DM) causes metabolic abnormalities and serious complications that have a profound impact on the patient's lifespan and quality of life. T2DM is mainly characterized by insulin resistance and hyperglycemia [3] Its common complications include microvascular disease (diabetic retinopathy, diabetic nephropathy, and diabetic neuropathy), macrovascular disease (diabetic heart disease, diabetic cerebrovascular disease, and peripheral vascular disease) $[4,5]$, and increased risk of cancer [6, 7]. At present, T2DM has a variety of therapeutic drugs, such as human insulin preparation, alpha glucosidase inhibitor, dipeptidyl peptidase- 4 inhibitor, incretin analogue, biguanide, insulin secretagogue, insulin sensitizer, and intestinal lipase inhibitor $[8,9]$. However, the currently used therapies have many side effects such as hypoglycemia, gastrointestinal problems, and weight gain [8]. Therefore, new drugs 
and natural compounds are constantly being tested to prevent and treat diabetes better [10].

Curcumin is a chemical component extracted from the rhizome of some plants. It has a series of effects such as blood lipid lowering, antitumor, anti-inflammatory, and antioxidation [11, 12] and has been used as a food flavoring agent, preservative, and ancillary medication for some diseases (such as heart disease and tumors) $[13,14]$. In the treatment of diabetes, there is also evidence to support curcumin as a part of the diabetes treatment program $[14,15]$. At present, many randomized controlled trials (RCTs) on the treatment of T2DM with curcumin have been published [11, 16-22], but there is still no systematic review and meta-analysis to assess the effects and safety of curcumin. Therefore, we decide to perform a systematic review and meta-analysis for the first time to evaluate the clinical effects of curcumin on T2DM.

\section{Materials and Methods}

2.1. Protocol. Study selection, assessment of eligibility criteria, data extraction, and statistical analyses were performed based on a predefined protocol registered on PROSPERO CRD42018089528 (see supplementary materials) [23].

2.2. Search Strategy and Selection Criteria. We searched the English database and the Chinese database from the beginning of their establishment to September 3, 2020. The English database includes EMBASE, Medline Complete, the Cochrane Library (until Issue 9, 2020), ClinicalTrials, PubMed, and Web of Science. The Chinese database includes the Chinese Science and Technology Periodical Database (VIP), Chinese Biomedical Database (CBM), Wan Fang Database (Chinese Ministry of Science and Technology), and China National Knowledge Infrastructure Databases (CNKI). The search strategy for PubMed is presented in Table 1 as an example.

Studies meeting the inclusion criteria would be included in this review: (1) participants: patients with type 2 diabetes mellitus; (2) intervention: curcumin with no limits on the type, dose, frequency, and so on; (3) comparisons: Western medicine, blanks, or placebo; (4) outcomes: primary outcomes: homeostasis model assessment-insulin resistance (HOMA-IR), glycosylated hemoglobin (HbAlc), total cholesterol (TC), and triglyceride (TG); secondary outcomes: body mass index (BMI), low-density lipoprotein cholesterol (LDL-C), high-density lipoprotein cholesterol (HDL-C), fasting glucose, and fasting insulin; (5) study type: randomized controlled trials (RCTs) with no limits on the manner by which randomization has been achieved on blinding or on the language of publication. Studies meeting the exclusion criteria would be excluded: (1) not T2DM patients; (2) the participant is not human; (3) nonoriginal research literature; and (4) non-RCT.

2.3. Data Extraction. Two reviewers independently extracted the data and it was checked by a third reviewer. When there is a disagreement, a consensus is reached through mutual discussion and negotiation with all reviewers. The extracted data include basic information (author, publication time, age of the research object, etc.), sample size, intervention measures, dose, intervention time, outcomes, etc. [24].

2.4. Study Quality Assessment. The risk of bias of RCTs was assessed by using the risk of bias assessment tool based on the Cochrane Handbook [25]. Two reviewers independently assessed the risk of bias. When there is a disagreement, a consensus is reached through mutual discussion and negotiation with all reviewers. The risk of bias is divided into three levels: high risk, low risk, and unclear. The content of the evaluation includes random sequence generation, allocation concealment, blinding, incomplete outcomes, selective reporting, and other bias.

2.5. Statistical Analysis. The data were analyzed by RevMan 5.3 software. Cochrane's $Q$ and $I^{2}$ test were used to judge the heterogeneity of different studies. If there is good homology between studies $\left(I^{2}<50 \%, P>0.1\right)$, the fixed-effects model is used for meta-analysis. If there is heterogeneity between studies $(I 2>50 \%, P<0.1)$, we first find the source of heterogeneity, conduct subgroup analysis, and then choose random-effects model or give up meta-analysis [26]. The dichotomous variable measure was summarized by risk ratio (RR) with a 95\% confidence interval (CI). The continuous outcomes underwent meta-analysis using weight mean differences (WMD) and 95\% CI. If the units of outcomes are different, or the value difference between RCTs is more than 10 times, the standard mean differences (SMD) and 95\% CI are used according to the situation.

2.6. Sensitivity Analysis and Publication Bias Detection. STATA 15.0 was utilized for sensitivity analysis and publication bias detection. Studies with RCTs $\geq 5$ were evaluated for publication bias. The outcomes with $P>0.1$ were thought to have publication bias. The outcomes that meet the following conditions are all subjected to sensitivity analysis: (1) random-effects model is used; (2) number of included RCTs $\geq 3$; and (3) the results of the fixed-effects model are inconsistent with the results of the random-effects model (whether it is a subgroup result or a summary result).

\section{Results}

3.1. Results of the Search. The total records identified through database searching and other sources were 431. Sixteen records were included after initial identification. Four records were excluded: Yang et al. is not RCT [27]; and the participants in the remaining 3 records are not only T2DM patients [28-30]. Eventually, 12 records were included to undergo analysis (Figure 1).

3.2. Description of Included Trials. Twelve records met the inclusion criteria $[11,16-22,31-34]$. Of the 12 records, 4 records [16-19] are from one RCT: Panahi et al., 2 records 
TABLE 1: Search strategy for PubMed.

\begin{tabular}{c} 
Database \\
\hline (Curcumin OR Turmeric Yellow OR Yellow, Turmeric OR Diferuloylmethane) AND (Type 2 diabetes mellitus OR Diabetes \\
Mellitus, Noninsulin-Dependent OR Diabetes Mellitus, Ketosis-Resistant OR Diabetes Mellitus, Ketosis Resistant OR Ketosis- \\
Resistant Diabetes Mellitus OR Diabetes Mellitus, Non Insulin Dependent OR Diabetes Mellitus, Non-Insulin-Dependent OR \\
Non-Insulin-Dependent Diabetes Mellitus OR Diabetes Mellitus, Stable OR Stable Diabetes Mellitus OR Diabetes Mellitus, Type \\
II OR NIDDM OR Diabetes Mellitus, Noninsulin Dependent OR Diabetes Mellitus, Maturity-Onset OR Diabetes Mellitus, \\
Maturity Onset OR Maturity-Onset Diabetes Mellitus OR Maturity Onset Diabetes Mellitus OR MODY OR Diabetes Mellitus, \\
Slow-Onset OR Diabetes Mellitus, Slow Onset OR Slow-Onset Diabetes Mellitus OR Type 2 Diabetes Mellitus OR Noninsulin- \\
Dependent Diabetes Mellitus OR Noninsulin Dependent Diabetes Mellitus OR Maturity-Onset Diabetes OR Diabetes, \\
Maturity-Onset OR Maturity Onset Diabetes OR Type 2 Diabetes OR Diabetes, Type 2 OR Diabetes Mellitus, Adult-Onset OR \\
Adult-Onset Diabetes Mellitus OR Diabetes Mellitus, Adult Onset) AND (randomized controlled trial [pt] OR controlled \\
clinical trial [pt] OR placebo [tiab] OR drug therapy [sh] OR trial [tiab] OR groups [tiab] OR clinical trials as topic [mesh: \\
noexp] OR Clinical Trial OR random* [tiab] OR random allocation [mh] OR single-blind method [mh] OR double-blind \\
method [mh] OR cross-over studies) NOT (animals [mh] NOT humans [mh])
\end{tabular}

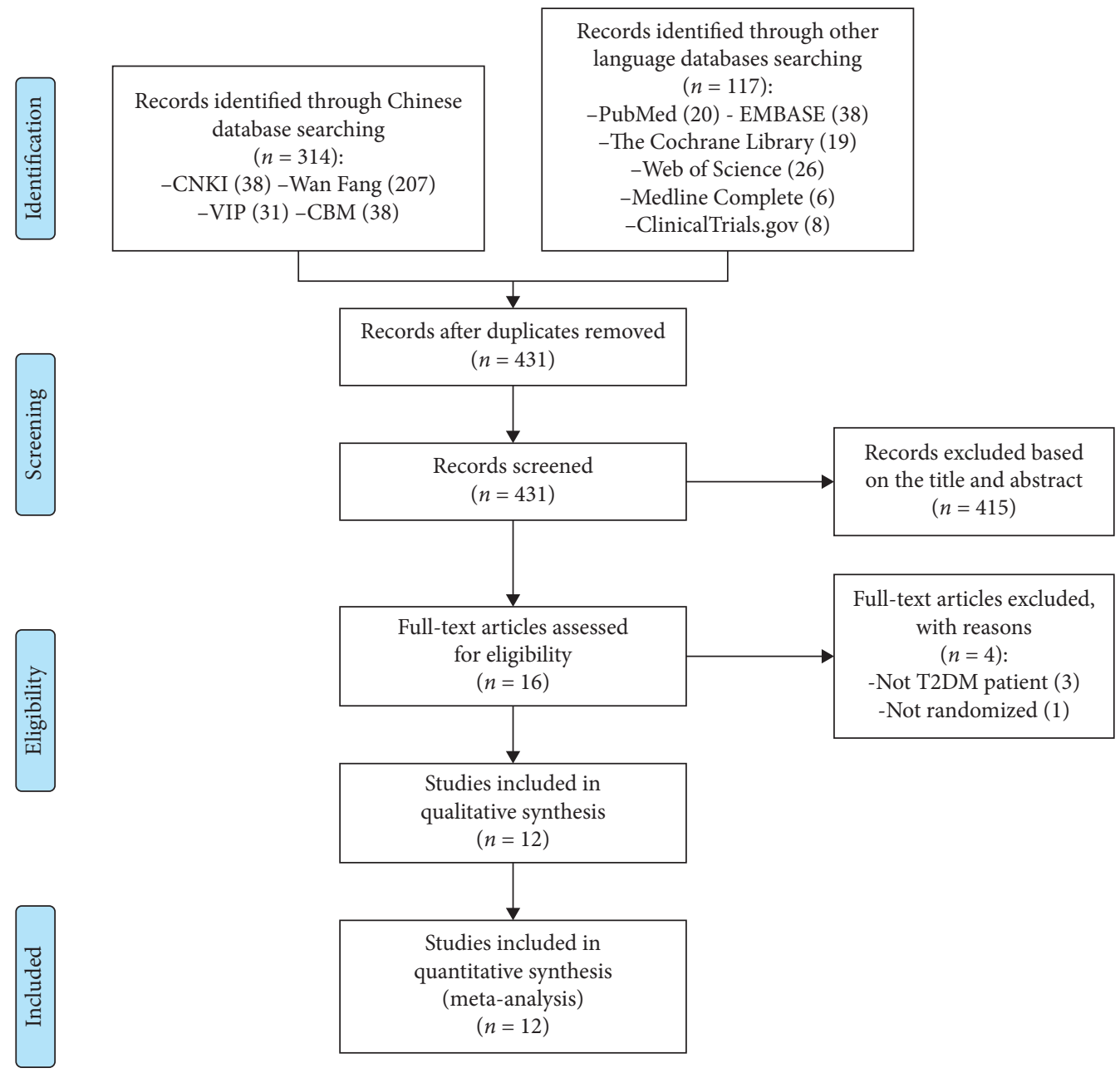

FIgURE 1: Flow diagram of searching and article selection.

$[20,21]$ are from the same RCT: $\mathrm{Na}$ et al, and 2 records $[31,32]$ are from one RCT: Asadi et al. Hence, a total of 7 RCTs are included in this review. All of them were parallelgroup RCTs. Study characteristics are presented in Table 2.

3.3. Risk of Bias of Included Studies. The summary and graph of risk of bias are shown in Figure 2.
3.3.1. Sequence Generation. Panahi et al. [16-19] and Khajehdehi et al. [22] failed to describe the method of randomization, and we therefore rated it as having an unclear risk of bias. $\mathrm{Na}$ et al. [20, 21], Chuengsamarn et al. [11], and Thota et al. [33] utilized a computer-generated random list, and Asadi et al. [31, 32] and Adibian et al. [34] utilized block randomization, so they were thought to have low risks of bias. 


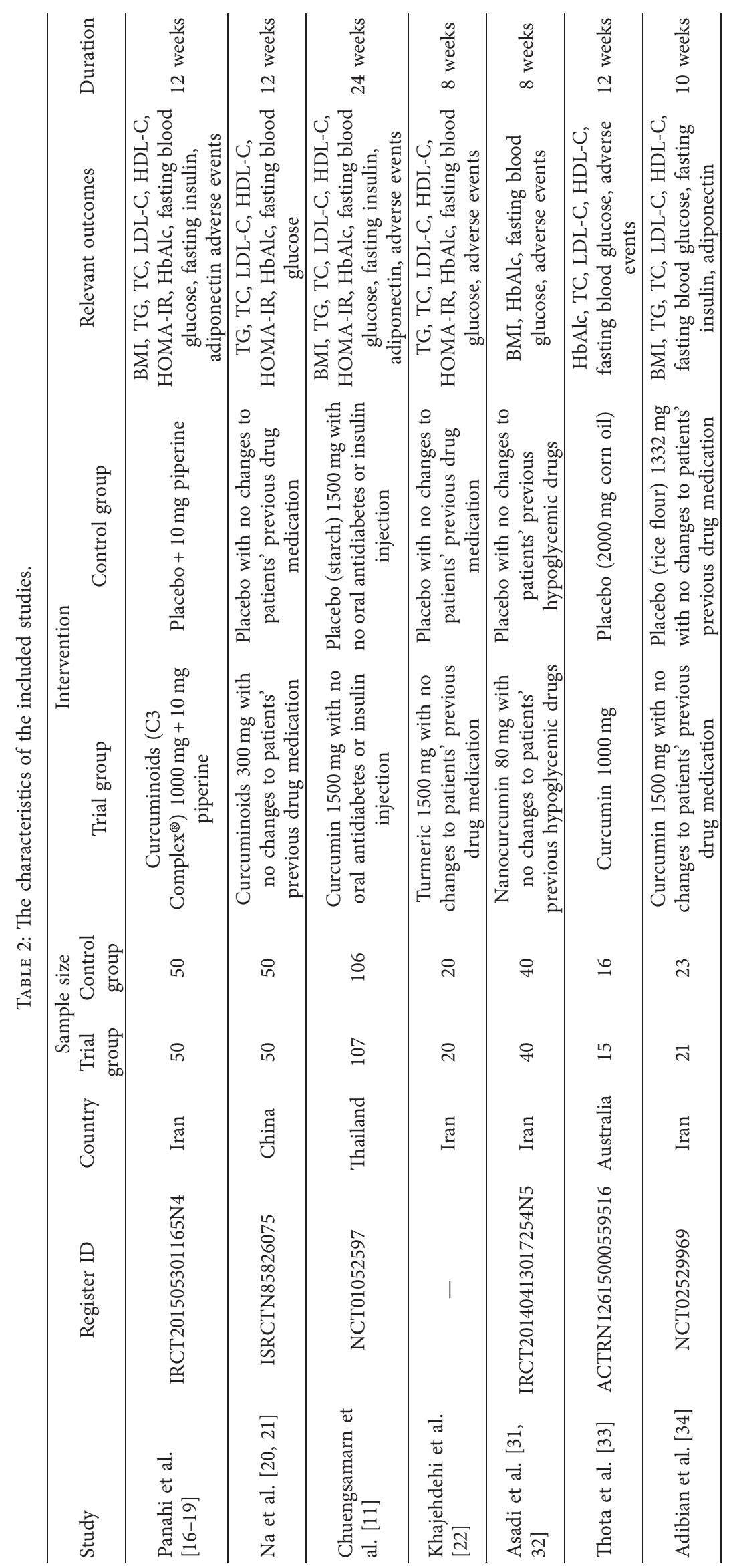




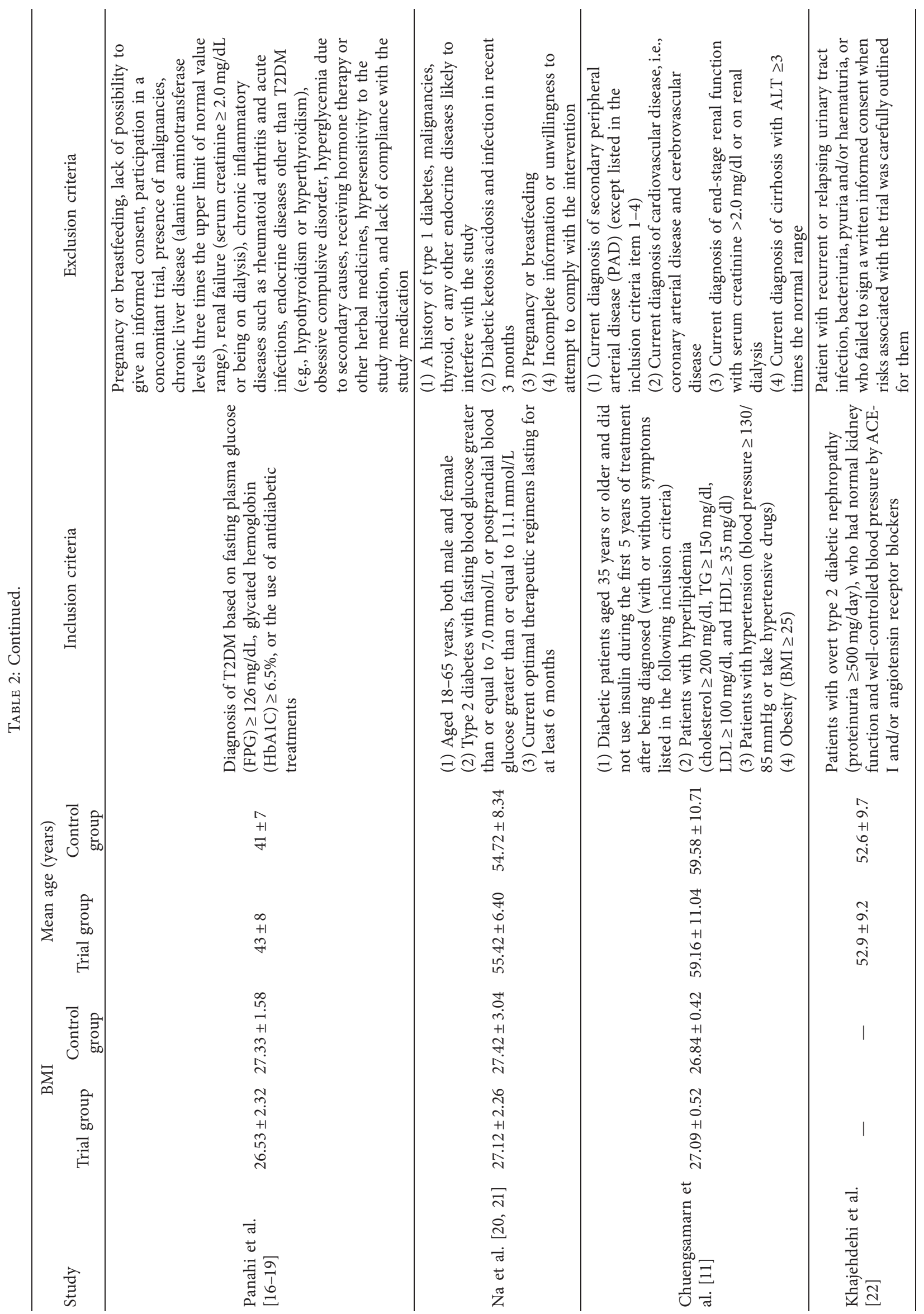




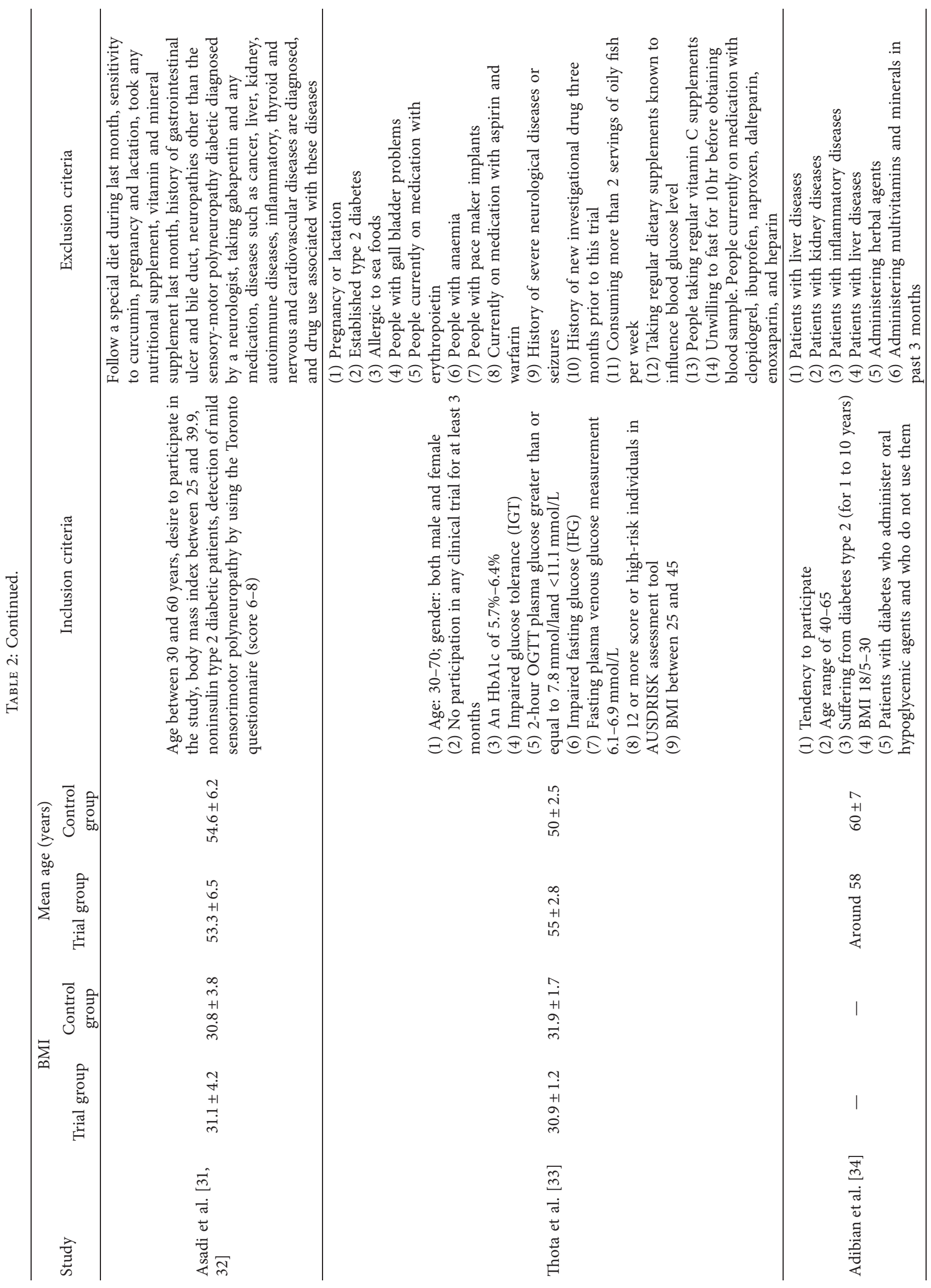




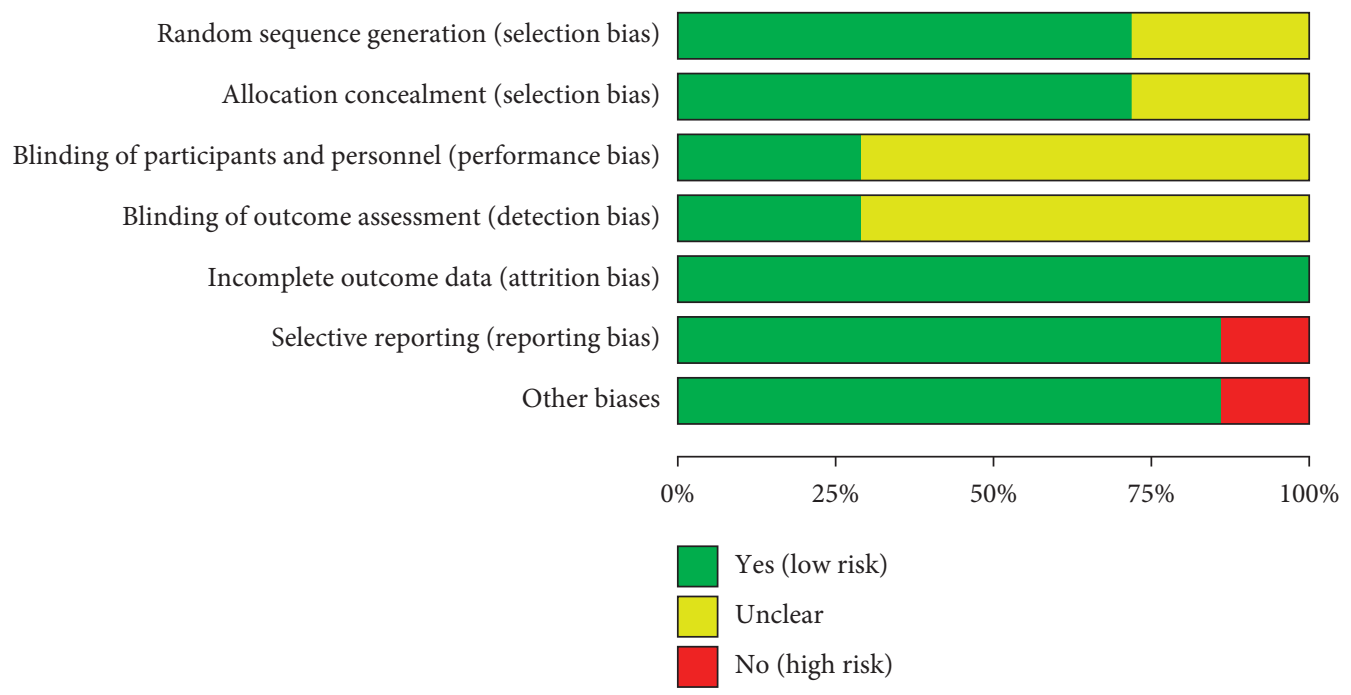

(a)

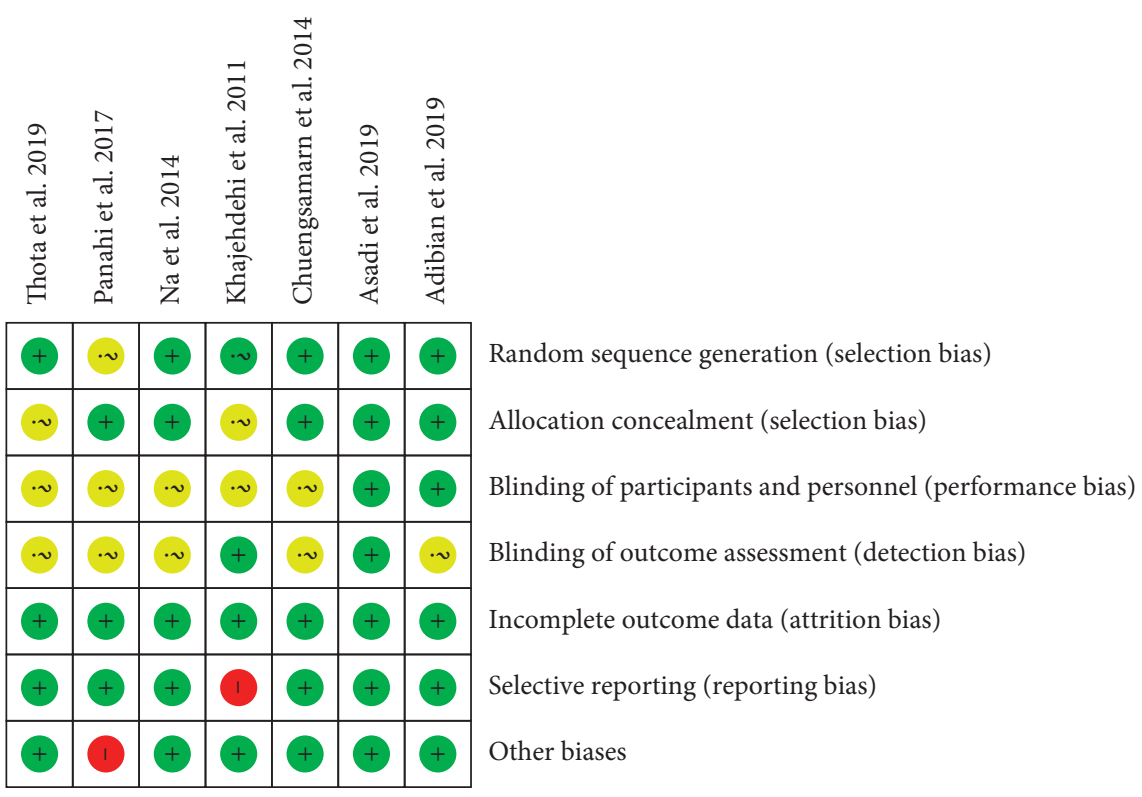

(b)

FiguRe 2: (a) Risk of bias graph. (b) Risk of bias summary.

3.3.2. Allocation Concealment. Khajehdehi et al. [22] and Thota et al. [33] did not describe an acceptable method of allocation concealment; therefore, they were rated as having an unclear risk of bias. Panahi et al. [16-19], Na et al. [20, 21], Asadi et al. [31, 32], and Adibian et al. [34] utilized the capsules in the same shape, size, and color to contain curcumin and placebo; Chuengsamarn et al. [11] used opaque and consecutively numbered envelopes. Hence, these five RCTs considered to have allocation concealment were rated as having low risks of bias.

3.3.3. Blinding, Incomplete Outcome Data, and Selective Reporting. All RCTs claimed to use blinding, but only Asadi et al. [31, 32] described the implementation process for researchers and participants, so only its double blinding was rated as a low risk of bias. Four RCTs [11, 16-21, 33] did not describe the implementation process for both researchers and participants, and they were rated as high risk of bias. Khajehdehi et al. [22] described blinding to researchers, so its blinding of outcome assessment (detection bias) was rated as low risk of bias. Adibian et al. [34] described blinding of participants, so the blinding of participants and personnel (performance bias) was rated as low risk of bias.

3.3.4. Incomplete Outcome Data and Selective Reporting. The incomplete outcome data of all RCTs are rated as low risk of bias because the number of missing persons and the reasons for the missing between groups is balanced. One 
RCT (Khajehdehi et al. [22]) failed to provide all outcomes mentioned in its protocols; thus, we thought its risk of bias was high. The others 6 RCTs reported study's prespecified outcomes that are of interest in the review; and their risks of bias were low.

3.3.5. Other Potential Bias. Panahi et al. [16-19] claimed that one of the authors had a conflict of interest and was therefore assessed as a high risk of bias. Other sources of bias in the other 6 RCTs were not found; therefore, the risks of other bias of the RCTs were low.

\subsection{Primary Outcomes}

3.4.1. Homeostasis Model Assessment-Insulin Resistance. Four RCTs [11, 16-21, 34] reported HOMA-IR. However, the data in Adibian et al. [34] cannot be extracted; hence, the data in four RCTs (207 participants in the curcumin group and 206 participants in the control group) were extracted. Due to the high heterogeneity, three RCTs were subdivided into two subgroups according to the region of the patients. After subdivision, the heterogeneity was still high in each subgroup (Asia Pacific: $I^{2}=86 \%, P=0.07$; the Middle East: not applicable) among the RCTs. The random-effects model was used. The HOMA-IR in the curcumin group was lower than that in the control group in the Middle East subgroup (WMD: -0.60 , 95\% CI: -0.74 to $-0.46, P<0.00001$ ) and the Asia subgroup (WMD: $-2.41,95 \%$ CI -4.44 to -0.39 , $P=0.02)$. However, the summary result showed that the difference in HOMA-IR between two groups was of no significance (WMD: $-1.76,95 \% \mathrm{CI}:-3.65$ to $0.13, P=0.07$ ) (Figure 3).

3.4.2. Glycosylated Hemoglobin. Six RCTs [11, 16-21, 31-34] reported HbAlc. However, the data in Adibian et al. [34] cannot be extracted; hence, the data in 5 RCTs including 262 participants in the curcumin group and 262 participants in the control group were extracted. Due to the high heterogeneity $\left(I^{2}=0 \%, P=0.96\right)$, the fixed-effect model was utilized. The results showed that, compared with the control group, curcumin can reduce HbAlc levels (WMD: -0.70 ; 95\% CI: $-0.87,-0.54 ; P<0.0001$ ) (Figure 4 ).

3.4.3. Total Cholesterol. Five RCTs [11, 16-22, 34] reported TC; however, Adibian et al. [34] used the same symbol for TC and TG, so it is not clear which group of data is TC, and the data cannot be extracted. The data in four RCTs containing 227 participants in the curcumin group and 226 participants in control group were extracted. Due to the high heterogeneity, four RCTs were subdivided into two subgroups according to the region of the patients. After subdivision, the heterogeneity was still high in each subgroup (Asia Pacific: $I^{2}=60 \%, P=0.11$; the Middle East: $I^{2}=71 \%$, $P=0.06)$ among the RCTs. The random-effects model was used. The difference between two groups in the Asia subgroup has statistical significance (WMD: $-23.45,95 \% \mathrm{CI}$ : -40.04 to $-6.84, P=0.006)$, but it did not have statistical significance in the Middle East subgroup (WMD: 22.91, 95\% CI: -16.94 to $62.75, P=0.26)$. The summary result also showed that the difference between two groups was of no significance (WMD: $-2.00,95 \%$ CI:-39.91 to 35.91, $P=0.92$ ) (Figure 5).

3.4.4. Triglyceride. Five RCTs $[11,16-22]$ reported TG; however, Adibian et al. [34] used the same symbol for TC and TG, so it is not clear which group of data is TG, and the data cannot be extracted. The data in four RCTs involving 227 participants in the curcumin group and 226 participants in the control group were extracted. Due to the high heterogeneity, four RCTs were subdivided into two subgroups according to the region of the patients. After subdivision, the heterogeneity was still high in the Asia subgroup $\left(I^{2}=80 \%, P=0.02\right)$ but low in the Middle East subgroup $\left(I^{2}=60 \%, P=0.11\right)$ among the RCTs. The random-effects model was used in the Asia subgroup. The difference between two groups in the Asia subgroup has statistical significance (WMD: $-54.14,95 \% \mathrm{CI}:-95.71$ to $-12.57, P=0.01$ ), but it did not have statistical significance in the Middle East subgroup (WMD -4.56, 95\% CI: -19.28 to $10.16, P=0.54)$. The summary result also showed that the difference between two groups was of no significance (WMD: $-33.45,95 \% \mathrm{CI}:-70.60$ to $3.71, P=0.08$ ) (Figure 6).

\subsection{Secondary Outcomes}

3.5.1. Body Mass Index. Three RCTs [11, 16-19, 31, 32] reported BMI. Due to the high heterogeneity $\left(I^{2}=89 \%\right.$, $P=0.0002$ ), the statistical analysis was abandoned according to the Cochrane Handbook for Systematic Reviews of Interventions. Panahi et al. [16-19] found that there was a statistically significant difference in BMI changes between the curcumin group and the control group $(-0.49 \pm 0.52$ versus $0.24 \pm 0.73 ; P<0.001)$. Chuengsamarn et al. [11] also found that there was a statistically significant difference in BMI changes between the curcumin group and the control group $(-1.97 \pm 5.38$ versus $0.16 \pm 4.32 ; P<0.001)$. However, Asadi et al. [31, 32] found that there were no significant differences in terms of BMI and weight between the two groups.

3.5.2. Fasting Blood Glucose and Fasting Insulin. All RCTs $[11,16-22,31-34]$ reported fasting blood glucose. However, the data in Adibian et al. [34] cannot be extracted; hence, the data in 6 RCTs include 282 participants in the curcumin group and 282 participants in the control group. Due to the high heterogeneity, 6 RCTs were subdivided into two subgroups according to the region of the patients. After subdivision, the heterogeneity was low in the Asia Pacific subgroup but high in the Middle East subgroup (Asia: 


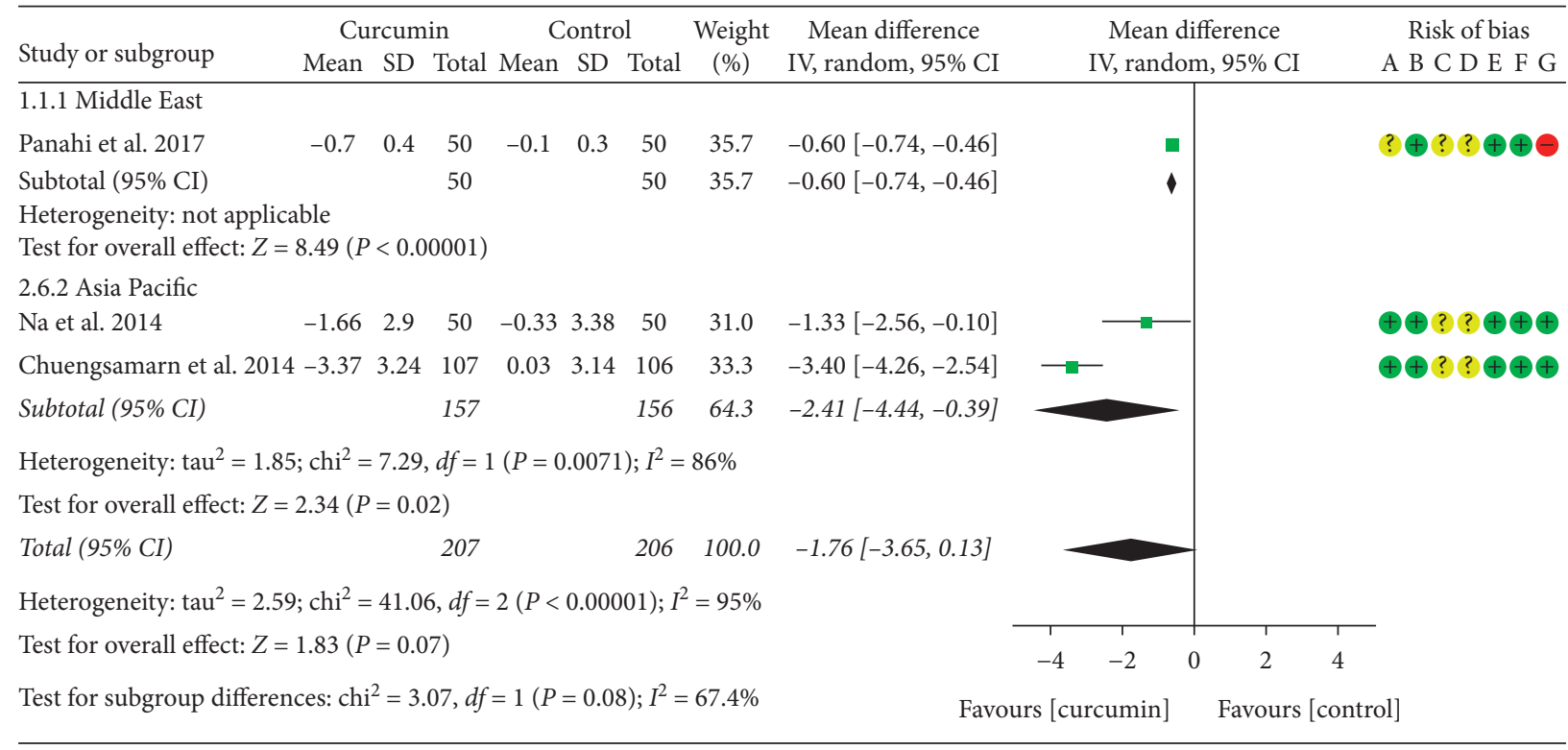

Risk of bias legend
(A) Random sequence generation (selection bias)
(B) Allocation concealment (selection bias)
(C) Blinding of participants and personnel (performance bias)
(D) Blinding of outcome assessment (detection bias)
(E) Incomplete outcome data (attrition bias)
(F) Selective reporting (reporting bias)
(G) Other biases

FIgURE 3: Homeostasis model assessment-insulin resistance.

$I^{2}=0 \%, P=0.79$; the Middle East: $I^{2}=74 \%, P=0.02$ ). The random-effects model was used. The fasting blood glucose in the curcumin group was lower than that in the control group Asia subgroup (SMD: $-0.57,95 \%$ CI: -0.79 to -0.36 , $P<0.00001)$. However, the difference between the curcumin group and the control group in the Middle East subgroup was of no statistical significance (SMD: 0.04, 95\% CI: -0.50 to $0.58, P=0.89$ ). The summary results also showed that the difference between two groups was of no statistical significance (SMD: $-0.28,95 \%$ CI: -0.62 to $0.06, P=0.11$ ) (Figure 7 ).

Two RCTs [11,16-19] reported fasting insulin. Due to the high heterogeneity $\left(I^{2}=96 \%, P<0.00001\right)$, the statistical analysis was abandoned according to the Cochrane Handbook for Systematic Reviews of Interventions. Panahi et al. [16-19] found no significant difference in fasting insulin levels between the two groups $(P>0.05)$, while Chuengsamarn et al. [11] found that the curcumin group had lower fasting insulin levels $(P<0.05)$.

3.5.3. Blood Lipid. Five RCTs [11, 16-22, 34] including 248 participants in the curcumin group and 249 participants in the control group reported LDL-C and HDL-C. Due to the high heterogeneity, 5RCTs were subdivided into two subgroups according to the region of the patients. After subdivision, the heterogeneity was low in each subgroup (Asia: $I^{2}=6 \%, P=0.30$; the Middle East: $I^{2}=29 \%, P=0.24$ ) among the RCTs. The fixed-effects model was used. The LDL-C in the curcumin group was lower than that in the control group in the Asia subgroup (WMD: -20.85 , 95\% CI: -28.78 to $-12.92, P<0.00001)$, but it was higher in the Middle East subgroup (WMD: 15.67, 95\% CI: 5.91 to 25.43, $P=0.002$ ) (Figure 8).

The RCTs in HDL-C were also subdivided into two subgroups according to the region of the patients for the same sake. After subdivision, the heterogeneity was still high in the two subgroups (Asia Pacific: $I^{2}=91 \%, P=0.001$; the Middle East: $I^{2}=76 \%, P=0.02$ ) among the RCTs. The random-effects model was used. The difference in HDL-C between two groups was of no statistical significance in each subgroup (the Middle East: WMD: -0.30 , 95\% CI: -3.78 to $3.19, P=0.87$; Asia: WMD: $6.01,95 \%$ CI: -2.58 to $14.60, P=0.17)$. The summary result also showed that the difference between two groups was of no statistical significance (WMD: 2.26, 95\% CI: -2.03 to $6.55, P=0.30$ ) (Figure 9). 


\begin{tabular}{|c|c|c|c|c|c|c|c|c|c|c|c|}
\hline \multirow{3}{*}{$\begin{array}{l}\text { Study or subgroup } \\
\text { Panahi et al. } 2017\end{array}$} & \multicolumn{3}{|c|}{ Curcumin } & \multicolumn{3}{|c|}{ Control } & \multirow{2}{*}{$\begin{array}{c}\text { Weight } \\
(\%)\end{array}$} & \multirow{2}{*}{$\begin{array}{l}\text { Mean difference } \\
\text { IV, fixed, 95\% CI }\end{array}$} & \multirow{2}{*}{\multicolumn{2}{|c|}{$\begin{array}{l}\text { Mean difference } \\
\text { IV, fixed, 95\% CI }\end{array}$}} & \multirow{3}{*}{$\begin{array}{c}\text { Risk of bias } \\
\text { A B C D E F G } \\
? \oplus ? ? \oplus \oplus \ominus\end{array}$} \\
\hline & \multirow{2}{*}{$\begin{array}{c}\text { Mean } \\
-0.9\end{array}$} & \multirow{2}{*}{$\begin{array}{l}\mathrm{SD} \\
1.1\end{array}$} & \multirow{2}{*}{$\begin{array}{c}\text { Total } \\
50\end{array}$} & \multirow{2}{*}{$\begin{array}{c}\text { Mean } \\
-0.2\end{array}$} & \multirow{2}{*}{$\begin{array}{l}\mathrm{SD} \\
0.5\end{array}$} & \multirow{2}{*}{$\begin{array}{c}\text { Total } \\
50\end{array}$} & & & & & \\
\hline & & & & & & & 25.2 & $-0.70[-1.03,-0.37]$ & -.- & & \\
\hline Na et al. 2014 & -0.75 & 1.94 & 50 & 0.27 & 2.57 & 50 & 3.6 & $-1.02[-1.91,-0.13]$ & & & $\oplus \oplus ? ?+\oplus \oplus$ \\
\hline Chuengsamarn et al.2014 & -0.64 & 1.12 & 107 & 0.01 & 1.13 & 106 & 31.0 & $-0.65[-0.95,-0.35]$ & - & & $\oplus \oplus ? ?+\oplus \oplus$ \\
\hline Asadi et al.2019 & -0.7 & 0.88 & 40 & 0.03 & 0.57 & 40 & 26.8 & $-0.70[-1.16,-0.24]$ & - & & $\oplus \oplus \oplus \oplus \oplus \oplus \oplus$ \\
\hline Thota et al.2019 & 1 & 0.7 & 15 & 1.7 & 0.6 & 16 & 13.4 & $-0.70[-1.16,-0.24]$ & - & & $\oplus$ ? ? ? $\oplus \oplus \oplus$ \\
\hline Total (95\% CI) & & & 262 & & & 262 & 100.0 & $-0.70[-0.87,-0.54]$ & $\diamond$ & & \\
\hline Heterogeneity: chi $^{2}=0.63$ & $d f=4$ & $(P=c$ & $0.96)$ & $I^{2}=0 \%$ & & & & & $\begin{array}{ll}-2 & -1\end{array}$ & 1 & \\
\hline Test for overall effect: $Z=$ & $8.20(P$ & $<0.0$ & 0001) & & & & & Favou & rcumin] & Favour & \\
\hline
\end{tabular}

Risk of bias legend
(A) Random sequence generation (selection bias)
(B) Allocation concealment (selection bias)
(C) Blinding of participants and personnel (performance bias)
(D) Blinding of outcome assessment (detection bias)
(E) Incomplete outcome data (attrition bias)
(F) Selective reporting (reporting bias)
(G) Other biases

Figure 4: Glycosylated hemoglobin.

3.5.4. Adiponectin. Three RCTs $[11,16-19,34]$ reported adiponectin. The data of Chuengsamarn et al. [11] could not be extracted, so only the data of 2 RCTs were extracted for analysis. The heterogeneity between RCTs is low $\left(I^{2}=0 \%\right.$, $P=0.49$ ), so a fixed-effects model is used. Compared with the control group, the curcumin group had higher adiponectin (SMD: $0.50,95 \%$ CI: 0.16 to $0.83, P=0.003$ ) (Figure 10).

3.6. Adverse Events. Four RCTs $[11,16-19,22,31,32]$ reported adverse events. Two RCTs [16-19, 22] showed that there are no severe related side effects in two groups. One RCT [11] showed that four patients in the curcumin group and four in the control group exhibited adverse events. Asadi et al. [31,32] reported 2 cases of stomach pain in the first few days, but did not specify the group.

3.7. Sensitivity Analysis Results. Sensitivity analysis was performed for 5 outcomes: HOMA-IR, TC, TG, FBG, and HDL-C. (1) In the outcome "HOMA-IR," after we omitted $\mathrm{Na}$ et al. $[20,21]$, we found that the estimate of the result moved out of the lower limit of 95\% CI (Figure 11(a)). (2) In the outcome "TC," after we omitted Panahi et al. [16-19], we found that the estimate of the result moved out of the lower limit of 95\% CI, while after omitting Chuengsamarn et al. [11], the estimate of the result moved out of the upper limit of $95 \%$ CI (Figure 11(b)). (3) In the outcome "TG," after omitting Chuengsamarn et al. [11], the estimate of the result moved out of the upper limit of 95\% CI (Figure 11(c)). (4) In the outcome "FBG," no matter which study was removed, the results were not significantly changed, suggesting that the heterogeneity may not come from RCT (Figure 11(d)). (5) In the outcome "HDL-C," after omitting Chuengsamarn et al. [11], the estimate of the result moved out of the lower limit of 95\% CI (Figure 11(e)). The abovementioned RCTs may be the source of heterogeneity of corresponding outcomes.

3.8. Publication Bias Detection. (1) HbAlc:the results of HbAlc indicate that there may be publication bias $(P=0.061)$ (Figure 12(a)). (2) FBG: the publication bias detection suggests that there may be no publication bias $(P=0.381)$ (Figure 12(b)). (3) LDL-C: the publication bias detection suggests that there may be no publication bias $(P=0.296)$ (Figure 12(c)). (4) HDL-C: the publication bias detection suggests that there may be no publication bias $(P=0.776)$ (Figure 12(d)).

\section{Discussion}

4.1. Main Findings. The HOMA-IR of the curcumin group is lower in Asia and the Middle East subgroups. The HbAlc in the curcumin group is lower than the control group. The TC, TG, and fasting blood glucose level of the curcumin group is lower in the Asia subgroup, while in the Middle East, the difference was of no statistical significance. For BMI, fasting insulin, and HDL-C level, there is no strong evidence that which one is better. Interestingly, although the LDL-C level of the curcumin group in the Asia subgroup is lower than that of the control group, the LDL-C level of the curcumin 


\begin{tabular}{|c|c|c|c|c|c|c|c|c|c|c|}
\hline \multirow{3}{*}{$\frac{\text { Study or subgroup }}{\text { 1.3.1 Middle East }}$} & \multicolumn{2}{|c|}{ Curcumin } & \multicolumn{3}{|c|}{ Control } & \multirow{2}{*}{$\begin{array}{l}\text { Weight } \\
(\%)\end{array}$} & \multirow{2}{*}{$\begin{array}{l}\text { Mean difference } \\
\text { IV, random, 95\% CI }\end{array}$} & \multirow{2}{*}{\multicolumn{2}{|c|}{$\begin{array}{l}\text { Mean difference } \\
\text { IV, random, 95\% CI }\end{array}$}} & \multirow{2}{*}{$\begin{array}{c}\text { Risk of bias } \\
\text { A B C D E F G }\end{array}$} \\
\hline & Mean SD & Total & Mean & $\mathrm{SD}$ & Total & & & & & \\
\hline & & & & & & & & & & \\
\hline Panahi et al. 2017 & 21.8625 .78 & 50 & -17.06 & 41.5 & 50 & 26.6 & $38.92[25.38,52.46]$ & & $=$ & $? \oplus ? ? \oplus \oplus \ominus$ \\
\hline Khajehdehi et al. 2011 & -26.774 .4 & 20 & -23.8 & 60.1 & 20 & 20.6 & $-2.90[44.82,39.02]$ & - & - & ? ? ? $\oplus \oplus \odot \oplus$ \\
\hline Subtotal (95\% CI) & & 70 & & & 70 & 47.2 & $22.91[-16.94,62.75]$ & & & \\
\hline \multicolumn{11}{|c|}{ Heterogeneity: $\operatorname{tau}^{2}=621.90 ; \mathrm{chi}^{2}=3.46, d f=1(P=0.06) ; I^{2}=71 \%$} \\
\hline \multicolumn{11}{|c|}{ Test for overall effect: $Z=1.13(P=0.26)$} \\
\hline \multicolumn{11}{|l|}{ 1.3.2 Asia Pacific } \\
\hline Na et al. 2014 & -20.542 .92 & 50 & -6.964 & 47.18 & 50 & 26.0 & $-13.54[-31.22,-4.14]$ & 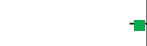 & & $\oplus \oplus ? ? ? \oplus \oplus$ \\
\hline Chuengsamarn et al. 2014 & -30.6342 .88 & 107 & 0.074 & 44.22 & 106 & 26.8 & $-30.70[-42.40,-19.00]$ & $=$ & & $\oplus \oplus ? ?+\oplus \oplus$ \\
\hline Subtotal (95\% CI) & & 157 & & & 156 & 52.8 & $-23.45[-40.06,-6.84]$ & $\diamond$ & & \\
\hline \multicolumn{11}{|c|}{ Heterogeneity: $\operatorname{tau}^{2}=88.74 ; \mathrm{chi}^{2}=2.52, d f=1(P=0.11) ; I^{2}=60 \%$} \\
\hline \multicolumn{11}{|c|}{ Test for overall effect: $Z=2.77(P=0.006)$} \\
\hline Total $(95 \%$ CI) & & 277 & & & 226 & 100.0 & $-2.00[-39.91,35.91]$ & & & \\
\hline \multicolumn{11}{|c|}{ Heterogeneity: tau $^{2}=1359.07 ; \mathrm{chi}^{2}=59.70, d f=3(P<0.00001) ; I^{2}=95 \%$} \\
\hline \multicolumn{8}{|c|}{ Test for overall effect: $Z=0.10(P=0.92)$} & $-200-100$ & $0 \quad 100200$ & \\
\hline \multicolumn{7}{|c|}{ Test for subgroup differences: $\mathrm{chi}^{2}=4.43, d f=1(P=0.04) ; I^{2}=77.4 \%$} & Favours & [curcumin] & Favours $[c$ & \\
\hline
\end{tabular}

Risk of bias legend
(A) Random sequence generation (selection bias)
(B) Allocation concealment (selection bias)
(C) Blinding of participants and personnel (performance bias)
(D) Blinding of outcome assessment (detection bias)
(E) Incomplete outcome data (attrition bias)
(F) Selective reporting (reporting bias)
(G) Other biases

Figure 5: Total cholesterol.

group was higher than the control group in the Middle east subgroup.

\subsection{Overall Completeness and Applicability of Evidence.} Most of RCTs come from Asia Pacific (especially Southeast Asia, like China and Thailand) and the Middle East (mainly Iran). Due to the lack of RCTs from all over the world, the applicability of the findings is limited.

4.3. Discussion of the Source of Heterogeneity. Most outcomes have heterogeneity, so this study conducted a sensitivity analysis to find the source of heterogeneity. Sensitivity analyses were performed for 5 outcomes: HOMA-IR, TC, TG, FBG, and HDL-C.

For outcome "HOMA-IR," compared with the other two RCTs, the dose of curcuminoids in $\mathrm{Na}$ et al. [20, 21] was lower (300 mg), while in Panahi et al. [16-19] it was $1000 \mathrm{mg}$, and in Chuengsamarn et al. [11] it was $1500 \mathrm{mg}$. Therefore, the heterogeneity of HOMA-IR may be related to the drug dose. For the outcome "TC," the mean age in Panahi et al. [16-19] is lower, which may be the source of heterogeneity.
For the outcome "TC," "TG," and "HDL-C," Chuengsamarn et al. [11] prepared curcumin monomer, while the preparations of several other RCTs are curcuminoids or turmeric, which suggests that different drug preparation methods may be the source of heterogeneity. For the outcome "FBG," no matter which study was removed, the results were not significantly changed, suggesting that the heterogeneity may not come from RCT.

In addition to the above possible sources of heterogeneity, heterogeneity may also come from ethnic differences, regional differences, gender differences, body size differences, and so on. More relevant RCTs are needed to be conducted in more diverse subgroups to reduce the heterogeneity of the research and stabilize the conclusions.

4.4. Novelty of This Research. This systematic review and meta-analysis showed that curcumin can improve HOMA-IR and HbAlc, especially in Asian patients and the Middle Eastern patients. In terms of blood lipids, curcumin can reduce TC, TG, and fasting blood glucose level in Asian patients, but does not improve in the Middle East patients significantly. Curcumin may lower the LDL-C 


\begin{tabular}{|c|c|c|c|c|c|c|c|c|c|c|c|}
\hline \multirow[b]{2}{*}{ Study or subgroup } & \multicolumn{3}{|c|}{ Curcumin } & \multicolumn{3}{|c|}{ Control } & \multirow{2}{*}{$\begin{array}{l}\text { Weight } \\
(\%)\end{array}$} & \multirow{2}{*}{$\begin{array}{l}\text { Mean difference } \\
\text { IV, random, 95\% CI }\end{array}$} & \multirow{2}{*}{\multicolumn{2}{|c|}{$\begin{array}{l}\text { Mean difference } \\
\text { IV, random, 95\% CI }\end{array}$}} & \multirow{2}{*}{$\begin{array}{l}\text { Risk of bias } \\
\text { A B C D E F G }\end{array}$} \\
\hline & Mean & SD & Total & Mean & SD & Total & & & & & \\
\hline \multicolumn{12}{|l|}{ 1.4.1 Middle East } \\
\hline Panahi et al. 2017 & -24.3 & 38.4 & 50 & -20.56 & 38.81 & 50 & 29.6 & $-3.74[-18.87,11.39]$ & & & ? $\odot ? ? \oplus \oplus \ominus$ \\
\hline Khajehdehi et al. 2011 & -39.5 & 103.9 & 20 & -20.5 & 101.1 & 20 & 16.3 & $-19.00[-82.53,44.53]$ & & - & ? ? ? $\oplus \oplus \ominus \oplus$ \\
\hline Subtotal (95\% CI) & & & 70 & & & 70 & 45.9 & $-4.56[-19.28,10.16]$ & & & \\
\hline \multicolumn{12}{|c|}{ Heterogeneity: $\operatorname{tau}^{2}=0.00 ; \mathrm{chi}^{2}=0.21, d f=1(P=0.65) ; I^{2}=0 \%$} \\
\hline \multicolumn{12}{|c|}{ Test for overall effect: $Z=0.61(P=0.54)$} \\
\hline \multicolumn{12}{|l|}{ 1.4.2 Asia Pacific } \\
\hline Na et al. 2014 & -39.85 & 48.7 & 50 & -7.08 & 82.35 & 50 & 26.9 & $-32.77[-59.29,-6.25]$ & - & & $\oplus \odot ? ? ? \oplus \oplus$ \\
\hline Chuengsamarn et al. 2014 & -75.26 & 88.17 & 107 & -0.07 & 101.31 & 106 & 27.2 & $-75.19[-100.71,-49.67]$ & $-\square$ & & $\oplus \odot ? ? ? \oplus \odot$ \\
\hline Subtotal (95\% CI) & & & 157 & & & 156 & 54.1 & $-54.14[-95.71,-12.57]$ & & & \\
\hline \multicolumn{12}{|c|}{ Heterogeneity: $\operatorname{tau}^{2}=723.46 ; \mathrm{chi}^{2}=5.10, d f=1(P=0.02) ; I^{2}=80 \%$} \\
\hline \multicolumn{12}{|c|}{ Test for overall effect: $Z=2.55(P=0.01)$} \\
\hline Total (95\% CI) & & & 227 & & & 226 & 100.0 & $-33.45[-70.60,3.71]$ & & & \\
\hline \multicolumn{12}{|c|}{ Heterogeneity: $\operatorname{tau}^{2}=1153.10 ; \mathrm{chi}^{2}=22.79, d f=3(P<0.0001) ; I^{2}=87 \%$} \\
\hline \multicolumn{8}{|c|}{ Test for overall effect: $Z=1.76(P=0.08)$} & -200 & -100 & 100 & 200 \\
\hline \multicolumn{8}{|c|}{ Test for subgroup differences: $\mathrm{chi}^{2}=4.86, d f=1(P=0.03) ; I^{2}=79.4 \%$} & \multicolumn{4}{|c|}{ Favours [control] } \\
\hline
\end{tabular}

Risk of bias legend
(A) Random sequence generation (selection bias)
(B) Allocation concealment (selection bias)
(C) Blinding of participants and personnel (performance bias)
(D) Blinding of outcome assessment (detection bias)
(E) Incomplete outcome data (attrition bias)
(F) Selective reporting (reporting bias)
(G) Other biases

Figure 6: Triglyceride.

level of Asian patients. Interestingly, after curcumin intervenes in the Middle Eastern patients, their LDL-C level has increased. This is something that needs attention in the future. Demmers et al. [35] estimated the available scientific data on the effectiveness and safety of medicinal food plants in the treatment of impaired glucose tolerance. It included an RCT of curcumin extract intervention for diabetes and found that the fasting blood glucose at 2 hours after intervention showed statistical significance after 3, 6, and 9 months $(P<0.01)$. In addition, the curcumin extract intervention (HbA1c) value showed statistical significance after 3,6 , and 9 months $(P<0.01)$. HOMA-IR after curcumin extract intervention showed statistical significance after 6 months and 9 months $(P<0.05$ and $P<0.01)$. It shows that curcumin has shown a reliable result that is effective in treating impaired glucose tolerance. Compared with Demmers et al.'s [35] research, our study is mainly devoted to exploring the interventional effects of curcumin and turmeric extracts on T2DM. We included more curcumin in the treatment of T2DM-related RCTs, and the evidence for improving the outcome of multiple related indicators of glucose tolerance is more sufficient. Compared to Marton et al.'s [36] review, (1) in this study, the RCT data (HOMA-IR, HbAlc, and so on) of curcumin treatment of T2DM were statistically analyzed (meta-analysis). (2) This study also conducted a subgroup analysis based on regions. (3) We adopted stricter screening criteria and merged the records belonging to the same RCT. (4) Sensitivity analysis was conducted in this study, which can more accurately locate the main source of heterogeneity. (5) The publication bias assessment was carried out in this study, and the publication bias of outcomes was excluded. In terms of improving metabolism, Roshanravan et al. [37] found the protective effect of supplementing Crocus sativus L. on hyperlipidemia and hyperglycemia through systematic reviews and meta-analysis studies. Our research also found that curcumin has the same clinical effect in improving the metabolism of diabetes.

4.5. The Strengths of This Review. This registered systematic review and meta-analysis is the first one that comprehensively evaluated the previous RCT of curcumin on T2DM and underwent a subgroup analysis to assess the applicable 


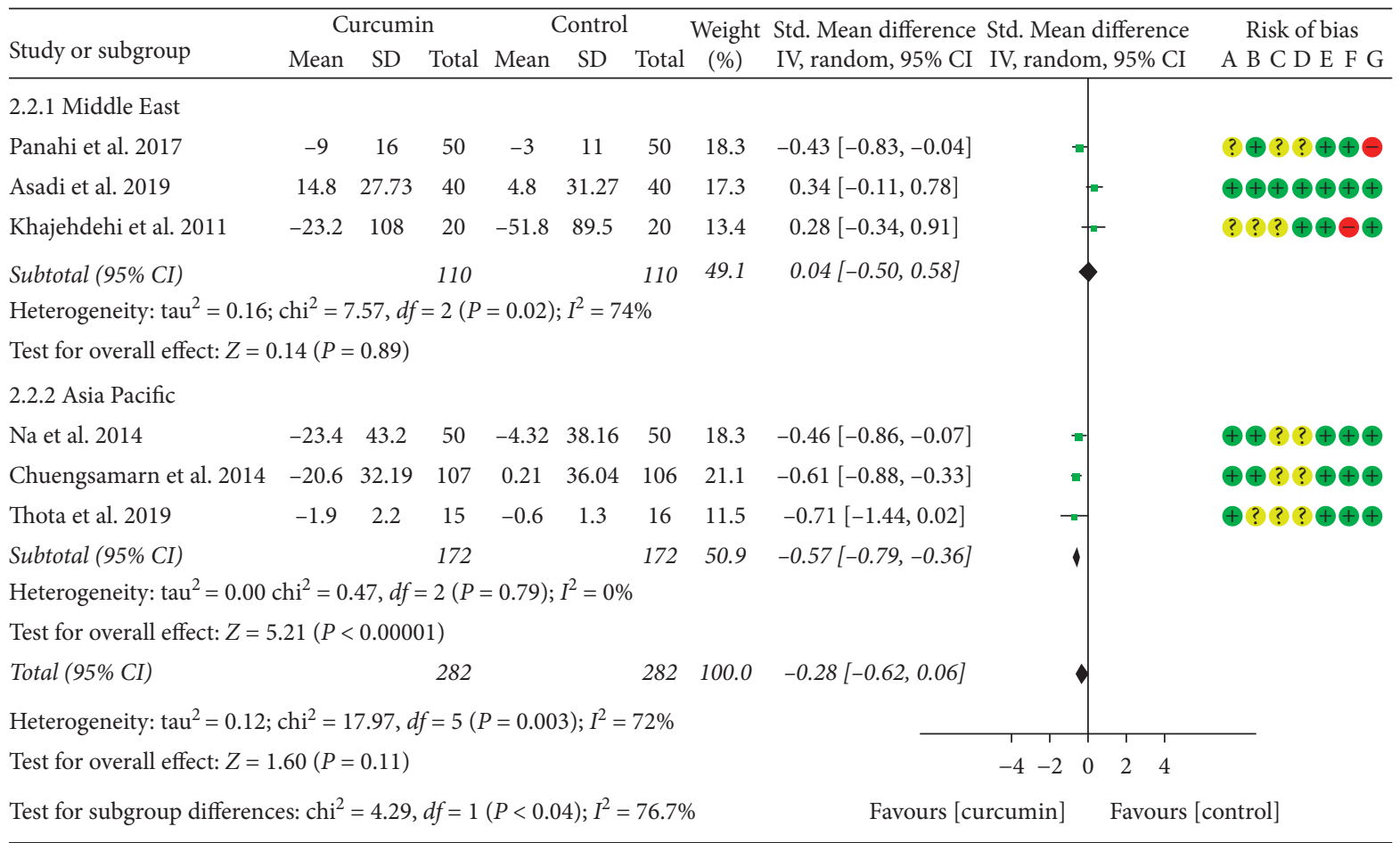

Risk of bias legend
(A) Random sequence generation (selection bias)
(B) Allocation concealment (selection bias)
(C) Blinding of participants and personnel (performance bias)
(D) Blinding of outcome assessment (detection bias)
(E) Incomplete outcome data (attrition bias)
(F) Selective reporting (reporting bias)
(G) Other biases

Figure 7: Fasting blood glucose.

population. It also collected the detailed data from each RCT and a comprehensive assessment of risk of bias was conducted.

4.6. The Limitations of This Review. RCTs from Asia and the Middle East account for a large proportion, which affects the applicability of the findings. The quantity and quality of RCTs are also not high in this review; several subgroups only include one RCT. Only 453 participants were included in this review, which may also impact the applicability of the findings. Meanwhile, half of RCTs [16-19, 22] have an unclear risk of bias in randomization, and one of the RCTs [22] has a high risk of bias in selective reporting; this may also influence the interpretation of the results. In addition, most of the outcomes have a high heterogeneity (such as HOMA-IR, TC, and TG) that cannot be eliminated by appropriate subgroup analysis. This also affected the applicability of the findings. The heterogeneity may come from the potential discrepancies in the pharmacological effects of various curcumin preparations, which may result from different standardizations of curcumin manufacturing process, dosage, duration of treatment, units of laboratory tests, and races of the selected patients or other places.

4.7. Implications for Future. This systematic review and meta-analysis found that curcumin may improve the HOMA-IR and fasting blood glucose of individuals in Asia 


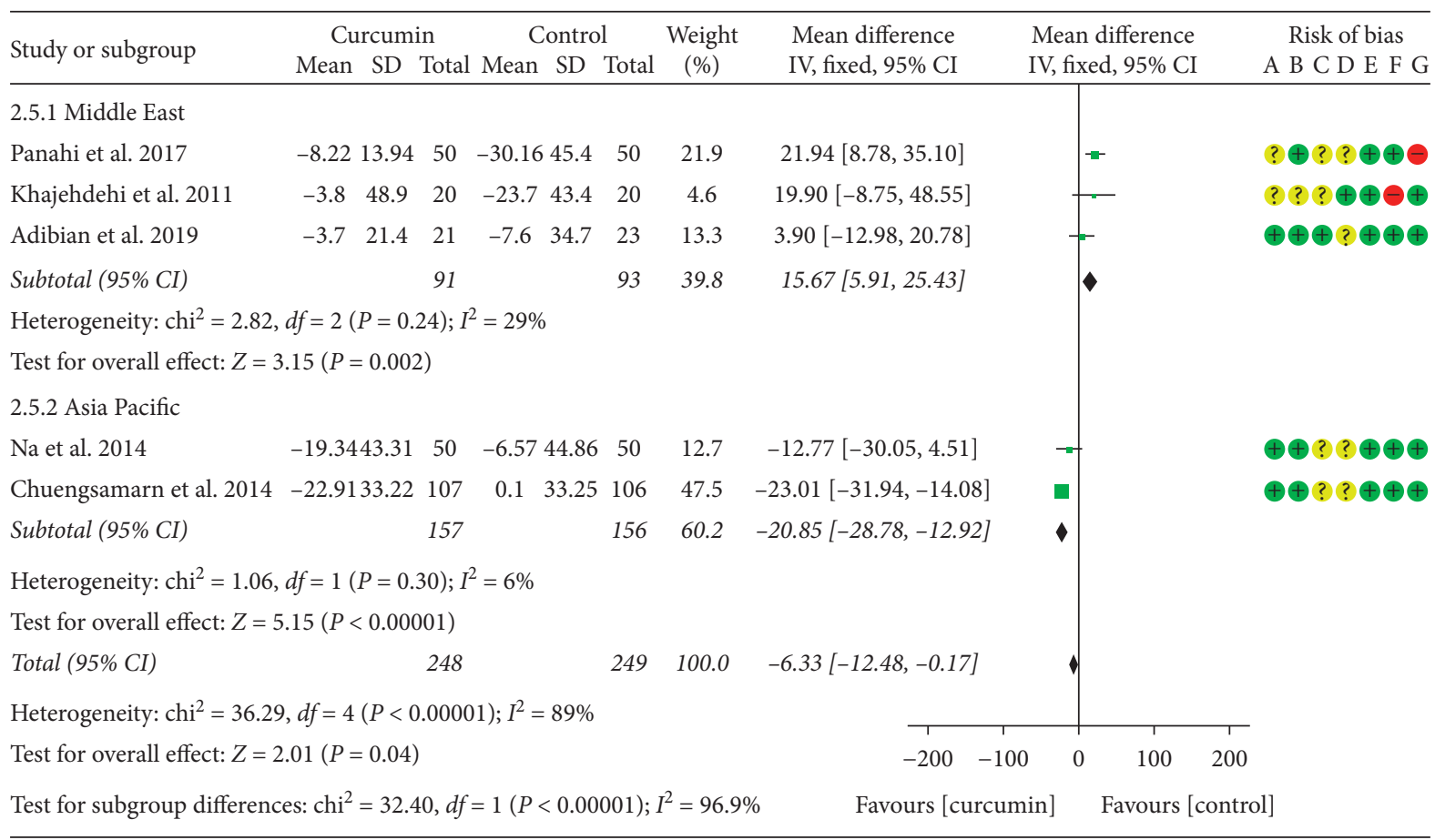

Risk of bias legend
(A) Random sequence generation (selection bias)
(B) Allocation concealment (selection bias)
(C) Blinding of participants and personnel (performance bias)
(D) Blinding of outcome assessment (detection bias)
(E) Incomplete outcome data (attrition bias)
(F) Selective reporting (reporting bias)
(G) Other biases

Figure 8: LDL-C.

and the Middle East. Curcumin may also improve the glycemic control in patients with T2DM. For blood lipids, curcumin may decrease the TC and TG in Asian T2DM patients, while its effects on the Middle Eastern T2DM patients may not be as significant as that on Asians; interestingly, according to the results, curcumin may decrease the LDL-C level in Asian T2DM patients, while it has the opposite effect on Middle Eastern patients. This may be related to ethnic differences, and more research is needed to amend or confirm it. In addition, in the aspect of decreasing BMI and fasting insulin, and improving HDL-C, curcumin may not have an advantage over the control group. However, due to the lack of evidence, more RCTs are needed. Last but not the least, for safety, there are no serious adverse events reported in RCTs, and the occurrence of adverse events in curcumin groups is the same as that of the placebo control group; therefore, it can be considered as a safety treatment based on current evidence.

In summary, curcumin may be more effective in Asia. In addition, for clinical practices, curcumin may be recommended as an adjunct to the treatment of T2DM patients to improve insulin resistance and glycemic control and reduce blood lipids. For Middle Eastern T2DM patients, the appropriate dosage, usage, and so on are yet to be confirmed. For future research, more RCTs about the adverse events and the T2DM-related outcomes are needed to revise or validate the findings in this review. The RCTs containing the data of the patients of other 


\begin{tabular}{|c|c|c|c|c|c|c|c|c|c|c|}
\hline \multirow{2}{*}{ Study or subgroup } & \multicolumn{3}{|c|}{ Curcumin } & \multicolumn{3}{|c|}{ Control } & \multirow{2}{*}{$\begin{array}{l}\text { Weight } \\
(\%)\end{array}$} & \multirow{2}{*}{$\begin{array}{c}\text { Mean difference } \\
\text { IV, random, } 95 \% \text { CI }\end{array}$} & \multirow{2}{*}{$\begin{array}{c}\text { Mean difference } \\
\text { IV, random, 95\% CI }\end{array}$} & \multirow{2}{*}{$\begin{array}{l}\text { Risk of bias } \\
\text { A B C D E F G }\end{array}$} \\
\hline & Mean & $\mathrm{SD}$ & Total & Mean & $\mathrm{SD}$ & Total & & & & \\
\hline \multicolumn{11}{|l|}{ 2.6.1 Middle East } \\
\hline Panahi et al. 2017 & 1.56 & 4.25 & 50 & -0.22 & 4.62 & 50 & 23.2 & $1.78[0.04,3.52]$ & 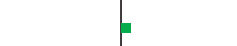 & ? $\odot ? ?+\oplus \ominus$ \\
\hline Khajehdehi et al. 2011 & -3.4 & 13.3 & 20 & -2.9 & 11.6 & 20 & 13.5 & $-0.50[-8.23,7.23]$ & - & ? ? ? $\oplus \oplus \ominus \oplus$ \\
\hline Adibian et al.2019 & -0.3 & 2.2 & 21 & -2.8 & 5.3 & 23 & 22.5 & $-2.50[-4.86,-0.14]$ & $=$ & $\oplus \oplus \oplus ? \oplus \oplus \oplus$ \\
\hline Subtotal (95\% CI) & & & 91 & & & 93 & 59.2 & $-0.30[-3.78,3.19]$ & $y$ & \\
\hline \multicolumn{11}{|c|}{ Heterogeneity: $\operatorname{tau}^{2}=6.29 ; \mathrm{chi}^{2}=8.22, d f=2(P=0.02) ; I^{2}=76 \%$} \\
\hline \multicolumn{11}{|c|}{ Test for overall effect: $Z=0.17(P=0.87)$} \\
\hline \multicolumn{11}{|l|}{ 2.6.2 Asia Pacific } \\
\hline Na et al. 2014 & 1.93 & 10.83 & 50 & 0.39 & 10.05 & 50 & 19.8 & $1.54[-2.56,5.64]$ & - & $\oplus \oplus ? ?+\oplus \oplus$ \\
\hline Chuengsamarn et al. 201 & 410.19 & 11.61 & 107 & -0.12 & 13.08 & 106 & 21.1 & $10.31[6.99,13.63]$ & $=$ & $\oplus \oplus ? ?+\oplus \oplus$ \\
\hline Subtotal (95\% CI) & & & 157 & & & 156 & 40.8 & $6.01[-2.58,14.60]$ & & \\
\hline \multicolumn{11}{|c|}{ Heterogeneity: $\mathrm{tau}^{2}=34.84 ; \mathrm{chi}^{2}=10.62, d f=1(P=0.001) ; I^{2}=91 \%$} \\
\hline \multicolumn{11}{|c|}{ Test for overall effect: $Z=1.37(P=0.17)$} \\
\hline Total $(95 \%$ CI) & & & 248 & & & 249 & 100.0 & $2.26[-2.03,6.55]$ & 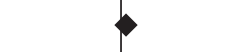 & \\
\hline \multicolumn{11}{|c|}{ Heterogeneity: $\operatorname{tau}^{2}=19.83 ; \mathrm{chi}^{2}=38.27, d f=4(P<0.00001) ; I^{2}=90 \%$} \\
\hline \multicolumn{11}{|c|}{ Test for overall effect: $Z=1.03(P=0.30)$} \\
\hline \multicolumn{11}{|c|}{ Test for subgroup differences: $\mathrm{chi}^{2}=1.78, d f=1(P=0.18) ; I^{2}=43.8 \%$} \\
\hline & & & & & & & & & $0-25$ & 50 \\
\hline \multicolumn{11}{|c|}{ Favours [curcumin] $\quad$ Favours [control] } \\
\hline
\end{tabular}

Risk of bias legend

(A) Random sequence generation (selection bias)

(B) Allocation concealment (selection bias)

(C) Blinding of participants and personnel (performance bias)

(D) Blinding of outcome assessment (detection bias)

(E) Incomplete outcome data (attrition bias)

(F) Selective reporting (reporting bias)

(G) Other biases

Figure 9: HDL-C.

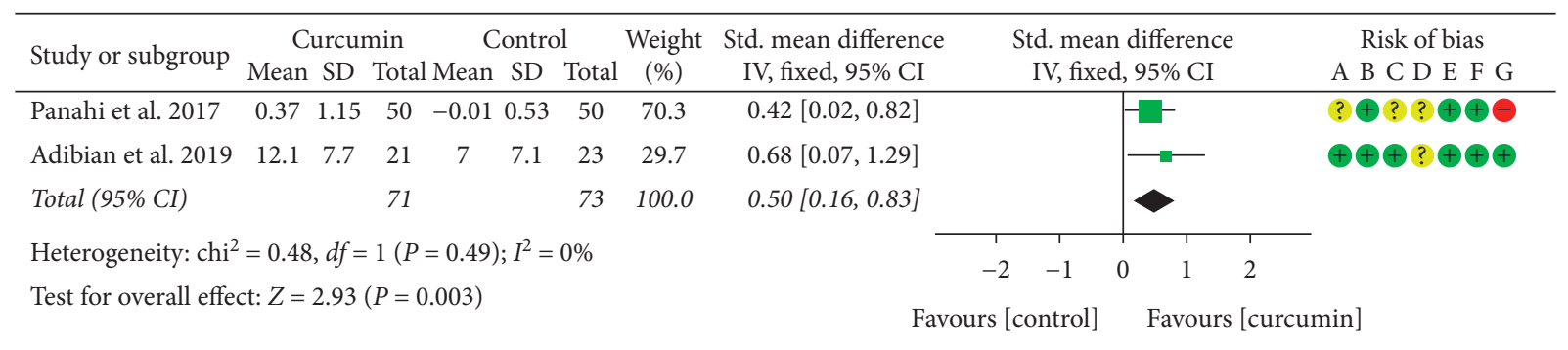

Risk of bias legend

(A) Random sequence generation (selection bias)

(B) Allocation concealment (selection bias)

(C) Blinding of participants and personnel (performance bias)

(D) Blinding of outcome assessment (detection bias)

(E) Incomplete outcome data (attrition bias)

(F) Selective reporting (reporting bias)

(G) Other biases

Figure 10: Adiponectin. 


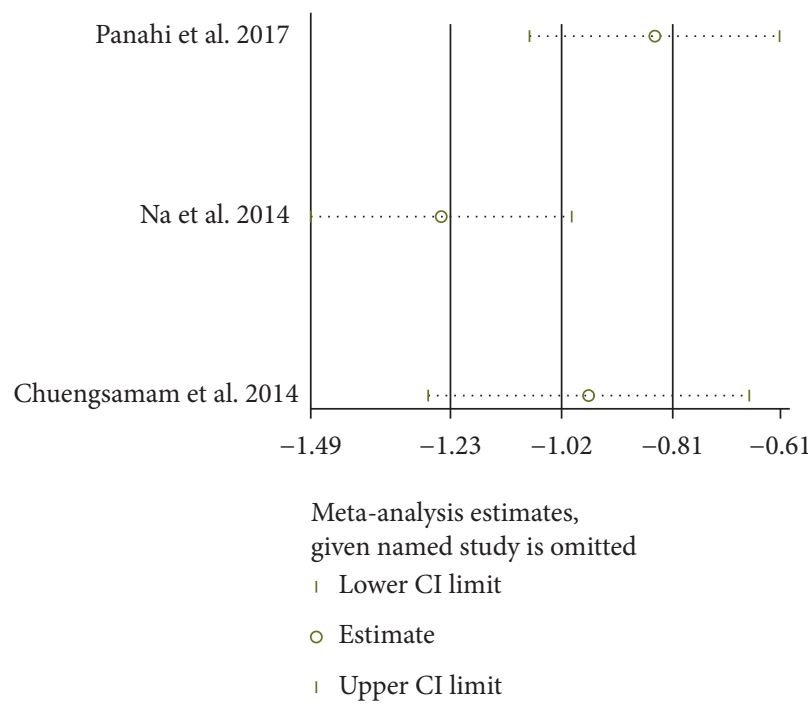

(a)

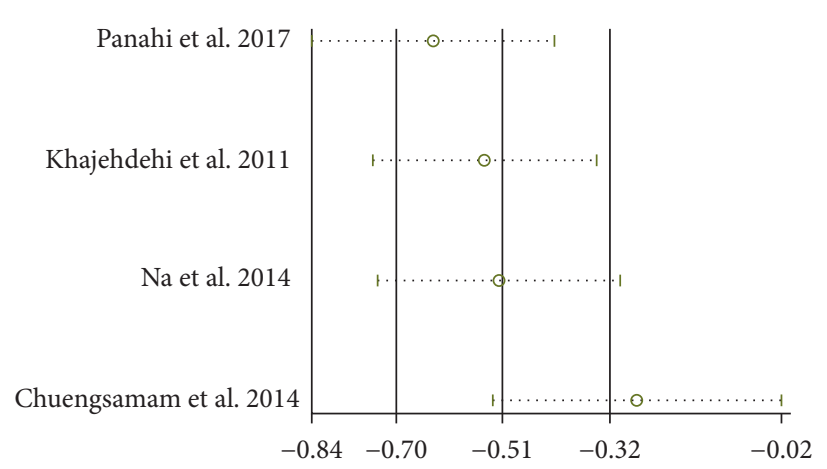

Meta-analysis estimates, given named study is omitted

I Lower CI limit

- Estimate

I Upper CI limit

(c)

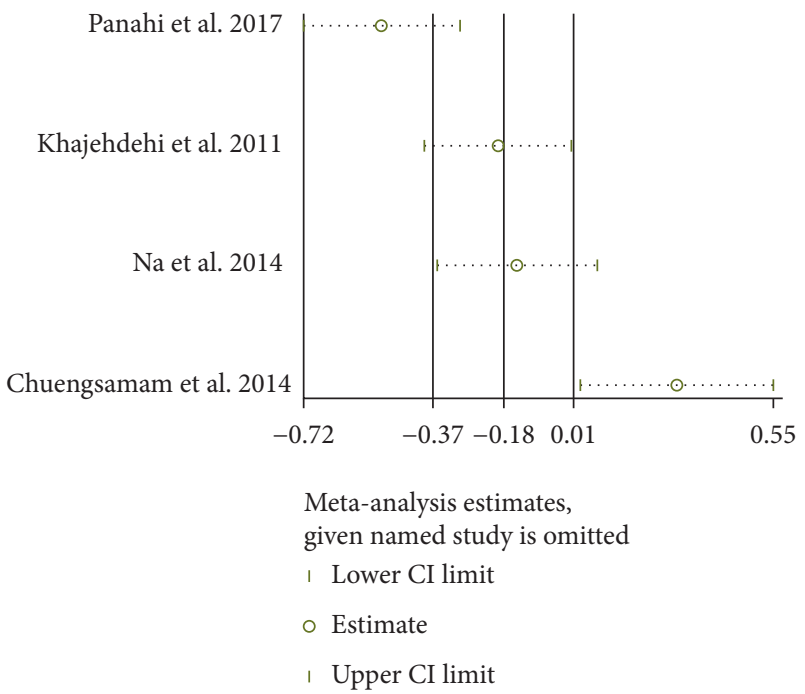

(b)

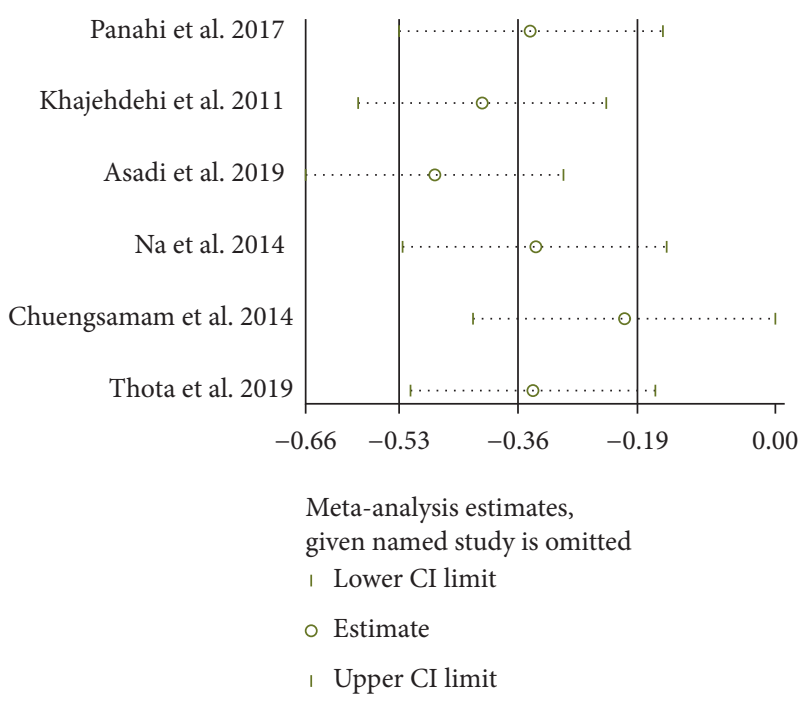

(d)

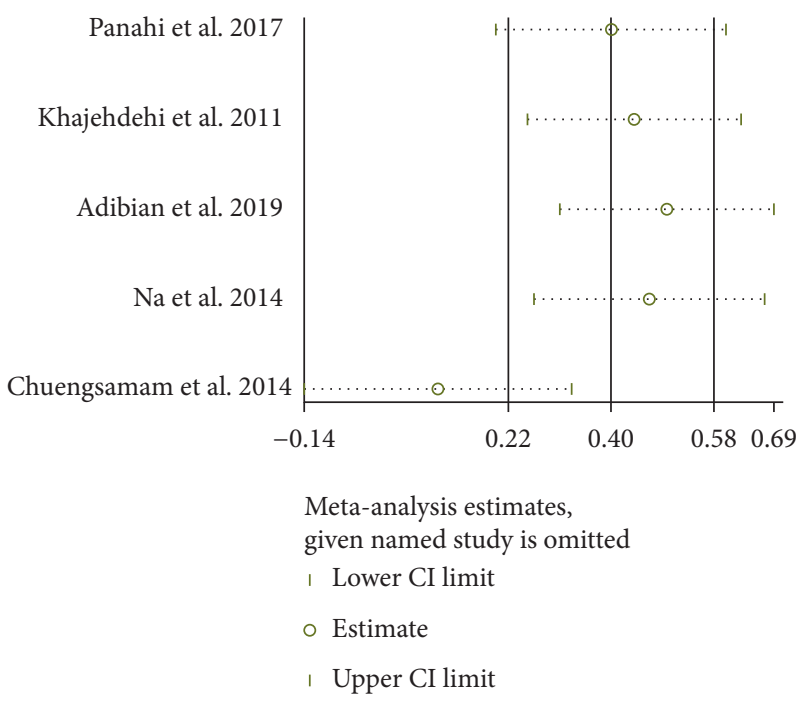

(e)

Figure 11: Sensitivity analysis results: (a) HOMA-IR; (b) TC; (c) TG; (d) FBG; (e) HDL-C. 

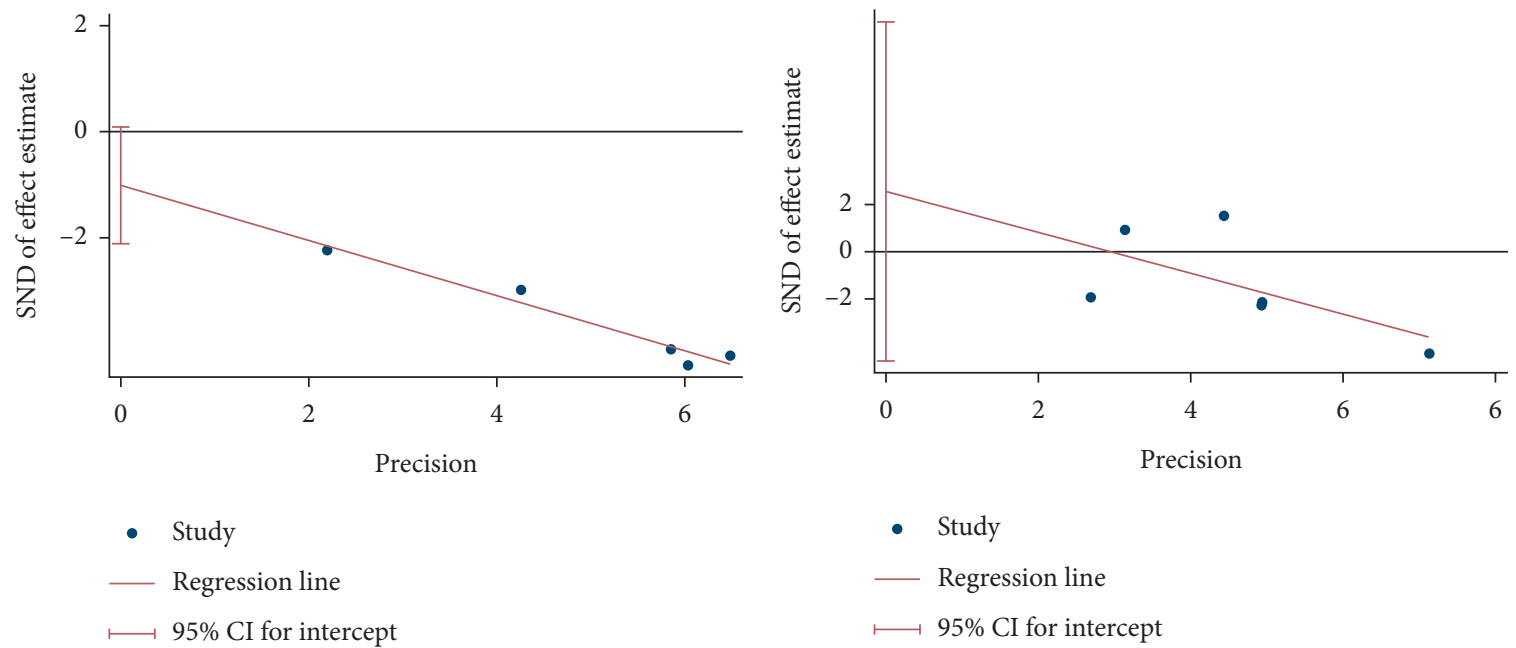

(a)
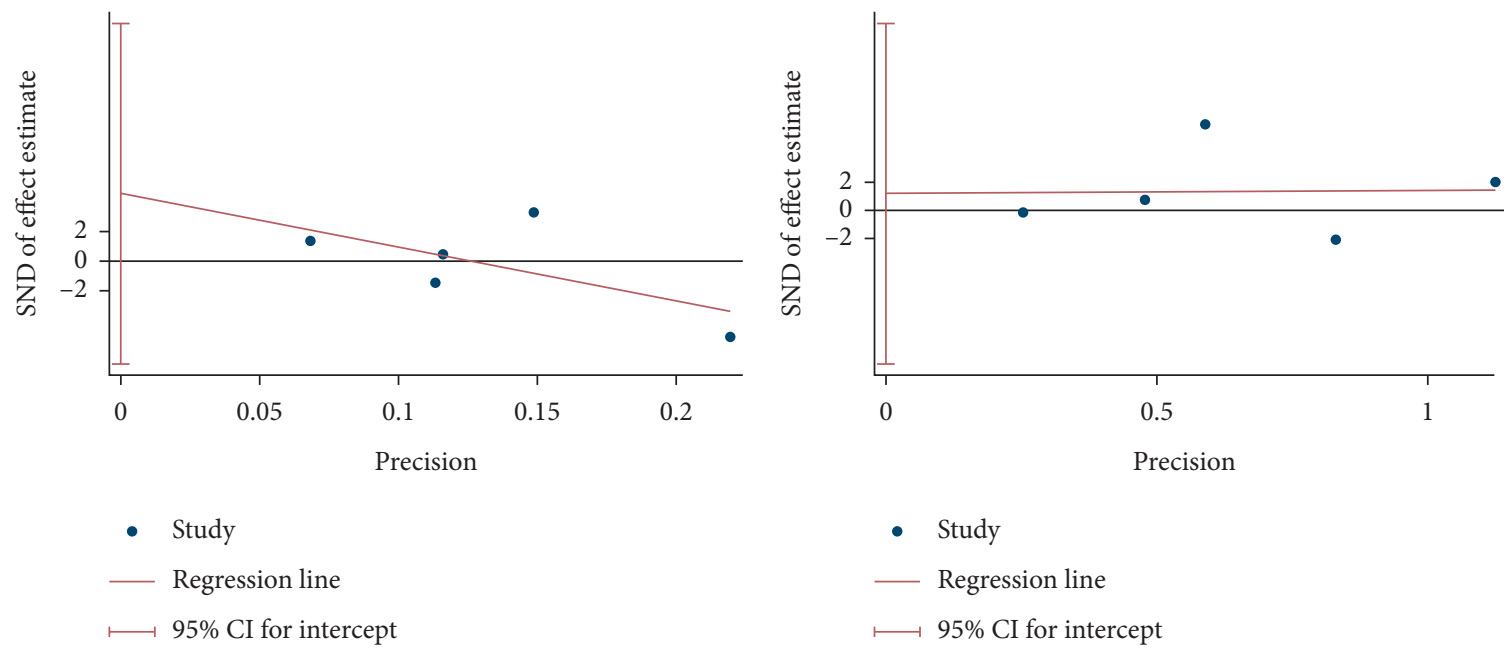

(c)

(d)

FIgURe 12: Publication bias detection

(a) $\mathrm{HbAlc}$;

(b) FBG; (c) LDL-C;

(d) HDL-C.

countries or regions around the world are also needed to expand the applicability of the results [38].

\section{Conclusion}

Based on the current evidence, curcumin may assist in improving insulin resistance, glycemic control, and decrease in TG and TC in patients with T2DM.

\section{Data Availability}

All data generated or analyzed during this study are included in this published article.

\section{Disclosure}

Tianqing Zhang and Qi He are co-first authors. Hengjing $\mathrm{Hu}$ is the corresponding author.

\section{Conflicts of Interest}

The authors declare that there are no conflicts of interest.

\section{Authors' Contributions}

Tianqing Zhang, Qi He, and Hengjing Hu are responsible for the study concept and design. Tianqing Zhang, Qi He, Yao Liu, Zhenrong Chen, and Hengjing $\mathrm{Hu}$ are responsible for the literature searching; Tianqing Zhang, Qi He, Yao Liu, Zhenrong Chen, and Henging $\mathrm{Hu}$ are responsible for data analysis and interpretation; Tianqing Zhang and Qi He drafted the paper; Hengjing $\mathrm{Hu}$ supervised the study; all authors participated in the analysis and interpretation of the data and approved the final manuscript.

\section{Acknowledgments}

This work was supported by grants from the National Natural Science Foundation of China (grant no. 81700306), the Natural Science Foundation of Hunan Province (grant no. 2018JJ3469), and the China Postdoctoral Science Foundation (grant no. 2017M622588). The authors thank Kailin Yang for his guidance on system review and metaanalysis. 


\section{Supplementary Materials}

"CRD42018089528" is the protocol of our systematic review and meta-analysis. The "PRISMA 2009 checklist" is a standardized checklist that make sure that systematic reviews and meta-analyses are implemented according to this standard. (Supplementary Materials)

\section{References}

[1] International Diabetes Federation, IDF Diabetes Atlas, International Diabetes Federation, Brussels, Belgium, 6th edition, 2013.

[2] GBD 2015 Mortality and Causes of Death Collaborators, "Global, regional, and national life expectancy, all-cause mortality, and cause-specific mortality for 249 causes of death, 1980-2015: a systematic analysis for the Global Burden of Disease Study 2015," Lancet, vol. 388, pp. 1459-1544, 2016.

[3] A. J. Vegas, O. Veiseh, M. Gürtler et al., "Long-term glycemic control using polymer-encapsulated human stem cell-derived beta cells in immune-competent mice," Nature Medicine, vol. 22, no. 3, pp. 306-311, 2016.

[4] J. M. Forbes and M. E. Cooper, "Mechanisms of diabetic complications," Physiological Reviews, vol. 93, no. 1, pp. 137-188, 2013.

[5] M. Stolar, "Glycemic control and complications in type 2 diabetes mellitus," The American Journal of Medicine, vol. 123, pp. S3-11, 2010.

[6] D. Wu, D. Hu, H. Chen et al., "Glucose-regulated phosphorylation of TET2 by AMPK reveals a pathway linking diabetes to cancer," Nature, vol. 559, no. 7715, pp. 637-641, 2018.

[7] J. Pearson-Stuttard, B. Zhou, V. Kontis, J. Bentham, M. J. Gunter, and M. Ezzati, "Worldwide burden of cancer attributable to diabetes and high body-mass index: a comparative risk assessment," The Lancet Diabetes \& Endocrinology, vol. 6, no. 6, pp. e6-e15, 2018.

[8] A. Y. Y. Cheng and I. G. Fantus, "Oral antihyperglycemic therapy for type 2 diabetes mellitus," Canadian Medical Association Journal, vol. 172, no. 2, pp. 213-226, 2005.

[9] G. Nicholson and G. M. Hall, "Diabetes mellitus: new drugs for a new epidemic," British Journal of Anaesthesia, vol. 107, no. 1, pp. 65-73, 2011.

[10] E. J. Verspohl, "Novel pharmacological approaches to the treatment of type 2 diabetes," Pharmacological Reviews, vol. 64, no. 2, pp. 188-237, 2012.

[11] S. Chuengsamarn, S. Rattanamongkolgul, B. Phonrat, R. Tungtrongchitr, and S. Jirawatnotai, "Reduction of atherogenic risk in patients with type 2 diabetes by curcuminoid extract: a randomized controlled trial," The Journal of $\mathrm{Nu}$ tritional Biochemistry, vol. 25, no. 2, pp. 144-150, 2014.

[12] V. Karuppagounder, S. Arumugam, V. V. Giridharan et al., "Tiny molecule, big power: multi-target approach for curcumin in diabetic cardiomyopathy," Nutrition, vol. 34, pp. 47-54, 2017.

[13] S. J. Hewlings and D. S. Kalman, "Curcumin: a review of its' effects on human health," Foods, vol. 22, no. 10, p. 6, 2017 pii: E92.

[14] J. Zheng, J. Cheng, S. Zheng, Q. Feng, and X. Xiao, "Curcumin, A polyphenolic curcuminoid with its protective effects and molecular mechanisms in diabetes and diabetic cardiomyopathy," Frontiers in Pharmacology, vol. 9, p. 472, 2018.

[15] N. Parsamanesh, M. Moossavi, A. Bahrami, A. E. Butler, and A. Sahebkar, "Therapeutic potential of curcumin in diabetic complications," Pharmacological Research, vol. 136, pp. 181193, 2018.

[16] Y. Panahi, N. Khalili, E. Sahebi et al., "Curcuminoids modify lipid profile in type 2 diabetes mellitus: a randomized controlled trial," Complementary Therapies in Medicine, vol. 33, pp. 1-5, 2017.

[17] Y. Panahi, N. Khalili, E. Sahebi et al., "Antioxidant effects of curcuminoids in patients with type 2 diabetes mellitus: a randomized controlled trial," Inflammopharmacology, vol. 25, no. 1, pp. 25-31, 2017.

[18] Y. Panahi, N. Khalili, E. Sahebi et al., "Curcuminoids plus piperine modulate adipokines in type 2 diabetes mellitus," Current Clinical Pharmacology, vol. 12, no. 4, pp. 253-258, 2018.

[19] Y. Panahi, N. Khalili, E. Sahebi et al., "Effects of curcuminoids plus piperine on glycemic, hepatic and inflammatory biomarkers in patients with type 2 diabetes mellitus: a randomized double-blind placebo-controlled trial," Drug Research, vol. 68, no. 07, pp. 403-409, 2018.

[20] L. X. Na, B. L. Yan, S. Jiang, H. L. Cui, Y. Li, and C. H. Sun, "Curcuminoids target decreasing serum adipocyte-fatty acid binding protein levels in their glucose-lowering effect in patients with type 2 diabetes," Biomedical and Environmental Sciences: BES, vol. 27, no. 11, pp. 902-906, 2014.

[21] L.-X. Na, Y. Li, H.-Z. Pan et al., "Curcuminoids exert glucoselowering effect in type 2 diabetes by decreasing serum free fatty acids: a double-blind, placebo-controlled trial," Molecular Nutrition \& Food Research, vol. 57, no. 9, pp. 1569-1577, 2013.

[22] P. Khajehdehi, M. Pakfetrat, K. Javidnia et al., "Oral supplementation of turmeric attenuates proteinuria, transforming growth factor- $\beta$ and interleukin- 8 levels in patients with overt type 2 diabetic nephropathy: a randomized, double-blind and placebo-controlled study," Scandinavian Journal of Urology and Nephrology, vol. 45, no. 5, pp. 365-370, 2011.

[23] D. Moher, A. Liberati, J. Tetzlaff, D. G. Altman, and The PRISMA Group, "Preferred reporting items for systematic reviews and meta-analyses: the PRISMA statement," Annals of Internal Medicine, vol. 151, no. 4, pp. 264-269, 2009.

[24] J. J. Deeks, J. P. Higgins, and D. G. Altman, "Chapter 16: special topics in statistics," in Cochrane Handbook for Systematic Reviews of Interventions, J. P. Higgins and S. Green, Eds., The Cochrane Collaboration, London, UK, 2011.

[25] J. J. Deeks, J. P. Higgins, and D. G. Altman, "Chapter 8: assessing risk of bias in included studies," in Cochrane Handbook or Systematic Reviews of Interventions Version 5.1.0, J. P. Higgins and S. Green, Eds., The Cochrane Collaboration, London, UK, 2011.

[26] J. J. Deeks, J. P. Higgins, and D. G. Altman, "Chapter 9: analyzing data and undertaking meta-analyses," in Cochrane Handbook for Systematic Reviews of Interventions, J. P. Higgins and S. Green, Eds., The Cochrane Collaboration, London, UK, 2011.

[27] H. Yang, W. Xu, Z. Zhou et al., "Curcumin attenuates urinary excretion of albumin in type II diabetic patients with enhancing nuclear factor erythroid-derived 2-like 2 (Nrf2) system and repressing inflammatory signaling efficacies," Experimental and Clinical Endocrinology \& Diabetes, vol. 123, no. 06, pp. 360-367, 2015.

[28] H. R. Rahimi, A. H. Mohammadpour, M Dastani et al., "The effect of nano-curcumin on $\mathrm{HbA1c}$, fasting blood glucose, and lipid profile in diabetic subjects: a randomized clinical trial," 
Avicenna Journal of Phytomedicine, vol. 6, no. 5, pp. 567-577, 2016.

[29] M. Funamoto, K. Shimizu, Y. Sunagawa et al., "Effects of highly absorbable curcumin in patients with impaired glucose tolerance and non-insulin-dependent diabetes mellitus," Journal of Diabetes Research, vol. 2019, 7 pages, 2019, PMC6906884, Article ID 8208237.

[30] R. N. Thota, J. I. Rosato, C. B. Dias, T. L. Burrows, R. N. Martins, and M. L. Garg, "Dietary supplementation with curcumin reduce circulating levels of glycogen synthase kinase- $3 \beta$ and islet amyloid polypeptide in adults with high risk of type 2 diabetes and alzheimer's disease," Nutrients, vol. 12, no. 4, p. 1032, 2020 PMID: 32283762.

[31] S. Asadi, M. S. Gholami, F. Siassi, M. Qorbani, and G. Sotoudeh, "Beneficial effects of nano-curcumin supplement on depression and anxiety in diabetic patients with peripheral neuropathy: a randomized, double-blind, placebocontrolled clinical trial," Phytotherapy Research, vol. 34, no. 4, pp. 896-903, 2020, PMID: 31788880.

[32] S. Asadi, M. S. Gholami, F. Siassi, M. Qorbani, K. Khamoshian, and G. Sotoudeh, "Nano curcumin supplementation reduced the severity of diabetic sensorimotor polyneuropathy in patients with type 2 diabetes mellitus: a randomized double-blind placebo- controlled clinical trial," Complementary Therapies in Medicine, vol. 43, pp. 253-260, 2019, PMID: 30935539.

[33] R. N. Thota, S. H. Acharya, and M. L. Garg, "Curcumin and/or omega-3 polyunsaturated fatty acids supplementation reduces insulin resistance and blood lipids in individuals with high risk of type 2 diabetes: a randomised controlled trial," Lipids in Health and Disease, vol. 18, no. 1, p. 31, 2019 PMID: 30684965.

[34] M. Adibian, H. Hodaei, O. Nikpayam, G. Sohrab, A. Hekmatdoost, and M. Hedayati, "The effects of curcumin supplementation on high-sensitivity C-reactive protein, serum adiponectin, and lipid profile in patients with type 2 diabetes: a randomized, double-blind, placebo-controlled trial," Phytotherapy Research, vol. 33, no. 5, pp. 1374-1383, 2019, Epub 2019 Mar 12. PMID: 30864188.

[35] A. Demmers, H. Korthout, F. S. Van Etten-Jamaludin, F. Kortekaas, and J. M. Maaskant, "Effects of medicinal food plants on impaired glucose tolerance: a systematic review of randomized controlled trials," Diabetes Research and Clinical Practice, vol. 131, pp. 91-106, 2017.

[36] L. T. Marton, L. M. Pescinini-E-Salzedas, M. E. C. Camargo et al., "The effects of curcumin on diabetes mellitus: a systematic review," Frontiers in Endocrinology, vol. 12, Article ID 669448, 2021.

[37] B. Roshanravan, S. Samarghandian, M. Ashrafizadeh, A. Amirabadizadeh, F. Saeedi, and T. Farkhondeh, "Metabolic impact of saffron and crocin: an updated systematic and metaanalysis of randomised clinical trials," Archives of Physiology and Biochemistry, vol. 2020, Article ID 1716020, 13 pages, 2020.

[38] S.-Y. Zhang, K.-L. Yang, L.-T. Zeng, X.-H. Wu, and H.-Y. Huang, "Effectiveness of coenzyme Q10 supplementation for type 2 diabetes mellitus: a systematic review and meta-analysis," International Journal of Endocrinology, vol. 2018, Article ID 6484839, 11 pages, 2018. 